\title{
Bezier Curves Based Numerical Solutions of Delay Systems with Inverse Time
}

\author{
F. Ghomanjani, ${ }^{1}$ M. H. Farahi, ${ }^{1}$ A. Kılıçman, ${ }^{2}$ A. V. Kamyad, ${ }^{1}$ and N. Pariz ${ }^{3}$ \\ ${ }^{1}$ Department of Mathematics, Ferdowsi University of Mashhad, Mashhad, Iran \\ ${ }^{2}$ Department of Mathematics, Universiti Putra Malaysia, 43400 Serdang, Selangor, Malaysia \\ ${ }^{3}$ Department of Control, Faculty of Engineering, Ferdowsi University of Mashhad, Mashhad, Iran
}

Correspondence should be addressed to A. Kılıçman; akilic@upm.edu.my

Received 10 July 2013; Accepted 29 December 2013; Published 27 February 2014

Academic Editor: Mufid Abudiab

Copyright @ 2014 F. Ghomanjani et al. This is an open access article distributed under the Creative Commons Attribution License, which permits unrestricted use, distribution, and reproduction in any medium, provided the original work is properly cited.

\begin{abstract}
This paper applied, for the first time, the Bernstein's approximation on delay differential equations and delay systems with inverse delay that models these problems. The direct algorithm is given for solving this problem. The delay function and inverse time function are expanded by the Bézier curves. The Bézier curves are chosen as piecewise polynomials of degree $n$, and the Bézier curves are determined on any subinterval by $n+1$ control points. The approximated solution of delay systems containing inverse time is derived. To validate accuracy of the present algorithm, some examples are solved.
\end{abstract}

\section{Introduction}

Delay differential equations (DDEs) differ from ODEs in that the derivative at any time depends on the solution at prior times (and in the case of neutral equations on the derivative at prior times).

DDEs often arise when traditional pointwise modeling assumptions are replaced by more realistic distributed assumptions, for example, when the birth rate of predators is affected by prior levels of predators or prey rather than by only the current levels in a predator-prey model.

Because the derivative $\dot{x}(t)$ depends on the solution at previous time(s), it is necessary to provide an initial history function to specify the value of the solution before time $t=$ 0 . In many common models the history is a constant; but nonconstant history functions are encountered routinely.

For most problems there is a jump derivative discontinuity at the initial time. In most models, the DDE and the initial function are incompatible: for some derivative order, usually the first, the left and right derivatives at $t=0$ are not equal. Delay systems containing inverse time are an important class of systems:

$$
\dot{x}(t)=x(t-1), \quad \dot{x}\left(0^{+}\right)=1, \quad \dot{x}\left(0^{-}\right)=0 .
$$

A fascinating property is how such derivative discontinuities are propagated in time. For the equation and history just described, for example, the initial first discontinuity is propagated as a second degree discontinuity at time $t=1$, as a third degree discontinuity at time $t=2$, and, more generally, as a discontinuity in the $(n+1)$ st derivative at time $t=n$.

Delay differential equations are type of differential equations where the time derivatives at the current time depend on the solution and possibly its derivatives at previous times (see [1-4]).

The basic theory concerning the stable factors, for example, existence and uniqueness of solutions, was presented in $[1,3]$. Since then, DDEs have been extensively studied in recent decades and a great number of monographs have been published including significant works on dynamics of DDEs by Hale and Lunel [5] and on stability by Niculescu [6]. The interest in study of DDEs is caused by the fact that many processes have time delays and have been models for better representations by systems of DDEs in science, engineering, economics, and so forth. Such systems, however, are still not feasible to actively analyze and control precisely; thus the study of systems of DDEs has actively been conducted over the recent decades (see [7-10]). 
Wu et al. [11] developed a computational method for solving an optimal control problem which is governed by a switched dynamical system with time delay. Kharatishivili [12] has approached this problem by extending Pontryagin's maximum principle to time delay systems. The actual solution involves a two-point boundary-value problem in which advances and delays are presented. In addition, this solution does not yield a feedback controller. Optimal-time control of delay systems has been considered by Oguztoreli [13] who obtained several results concerning bang-bang controls which are parallel to those of LaSalle [14] for nondelay systems. For a time-invariant system with an infinite upper limit in the performance measure, Krasovskii [15] has developed the forms of the controller and the performance measure. Ross [16] has obtained a set of differential equations for the unknowns in the forms of Krasovskii. However, Ross's results are not applicable to time-varying systems with a finite limit in the performance measure.

Basin and Perez [17] presented an optimal regulator for a linear system with multiple state and input delays and a quadratic criterion. The optimal regulator equations were obtained by reducing the original problem to the linearquadratic regulator design for a system without delays (see $[17,18])$.

This paper aims at solving delay systems containing inverse time of the following form:

$$
\begin{gathered}
\dot{\mathbf{x}}(t)=A(t) \mathbf{x}(t)+C(t)\left(x_{1}\left(t-\tau_{1}\right) \cdots x_{p}\left(t-\tau_{p}\right)\right)^{T} \\
+D(t)\left(x_{1}\left(t_{f}-t\right) \cdots x_{p}\left(t_{f}-t\right)\right)^{T}+G(t) \mathbf{u}(t) \\
\mathbf{x}(t)=\phi(t), \quad t \in\left[-\tau_{\max }, t_{0}\right]
\end{gathered}
$$

where $\mathbf{x}(t)=\left(x_{1}(t) \cdots x_{p}(t)\right)^{T} \in \mathbf{R}^{p}, \mathbf{u}(t)=\left(u_{1}(t) \cdots\right.$ $\left.u_{m}(t)\right)^{T} \in \mathbf{R}^{m}$ are, respectively, state and control functions while $\phi(t)=\left(\phi_{1}(t) \cdots \phi_{p}(t)\right)^{T}$ is known vector function and $\tau_{i}$ 's $(i=1,2, \ldots, p)$ are nonnegative constant time delays, and $\tau_{\max }=\max \left\{\tau_{i}, 1 \leq i \leq p\right\}$. We assume the matrices $A(t)=\left[a_{i j}(t)\right]_{p \times p}, C(t)=\left[c_{i j}(t)\right]_{p \times p}, D(t)=\left[d_{i j}(t)\right]_{p \times p}$, and $G(t)=\left[g_{i j}(t)\right]_{m \times m}$ are matrix functions. We need to impose the continuity condition on $\mathbf{x}(t)$ and its first derivative where these constraints appeared in Section 2.

Piecewise polynomial functions are often used to represent the approximate solution in the numerical solution of differential equations (see [19-22]). B-splines, due to numerical stability and arbitrary order of accuracy, have become popular tools for solving differential equations (where Bézier form is a special case of B-splines). There are many papers and books dealing with the Bézier curves or surface techniques.

Harada and Nakamae [23], Nürnberger and Zeilfelder [24] used the Bézier control points in approximated data and functions. Zheng et al. [22] proposed the use of control points of the Bernstein-Bézier form for solving differential equations numerically and also Evrenosoglu and Somali [25] used this approach for solving singular perturbed two-point boundary-value problems. The Bézier curves are used in solving partial differential equations; as well, Wave and Heat equations are solved in Bézier form (see [26-29]), the Bézier curves are used for solving dynamical systems (see [30]), and also the Bézier control points method is used for solving delay differential equation (see $[31,32]$ ).

The Bézier curves method was presented which was stated for solving the optimal control systems with pantograph delays (see [33]). The method was computationally attractive and also reduced the CPU time and the computer memory and at the same time keeps the accuracy of the solution. The algorithm had been successfully applied to the pantograph equations. Comparing with other methods, the results of numerical examples demonstrated that this method was more accurate than some existing methods (see [33]).

Using Bezier curve, Ghomanjani et al. [34] had used least square method for numerical solutions of time-varying linear optimal control problems with time delays in state and control.

Some other applications of the Bézier functions and control points are found in [35-37] that are used in computer aided geometric design and image compression.

The use of the Bézier curves is a novel idea for solving delay systems containing inverse time. The approach used in this paper reduces the CPU time and the computer memory comparing with existing methods (see the numerical results). Although the method is very easy to use and straightforward, the obtained results are satisfactory (see the numerical results). We suggest a technique similar to the one used in $[22,25]$ for solving delay systems containing inverse time. The current paper is organized as follows.

In Section 2, Function approximation will be introduced. Convergence analysis will be stated in Section 3. In Section 4, some numerical examples are solved which show the efficiency and reliability of the method. Finally, Section 5 will give a conclusion briefly.

\section{Function Approximation}

Divide the interval $\left[t_{0}, t_{f}\right]$ into a set of grid points such that

$$
t_{i}=t_{0}+i h, \quad i=0,1, \ldots, k
$$

where $h=\left(t_{f}-t_{0}\right) / k$ and $k$ is a positive integer. Let $S_{j}=$ $\left[t_{j-1}, t_{j}\right]$ for $j=1,2, \ldots, k$. Then, for $t \in S_{j}$, delay systems containing inverse time (2) can be decomposed to the following problem:

$$
\begin{aligned}
\dot{\mathbf{x}}_{j}(t)= & A(t) \mathbf{x}_{j}(t) \\
& +C(t)\left(x_{1}^{-k_{1}^{1}+j}\left(t-\tau_{1}\right) \cdots x_{p}^{-k_{1}^{p}+j}\left(t-\tau_{p}\right)\right)^{T} \\
& +D(t)\left(x_{1}^{k_{2}-j}\left(t_{f}-t\right) \cdots x_{p}^{k_{2}-j}\left(t_{f}-t\right)\right)^{T} \\
& +G(t) \mathbf{u}_{j}(t), \\
& \mathbf{x}_{j}(\theta)=\phi(\theta), \quad \theta \in\left[-\tau_{\max }, t_{0}\right]
\end{aligned}
$$

where $\mathbf{x}_{j}(t)=\left(x_{1}^{j}(t) \cdots x_{p}^{j}(t)\right)^{T}$ and $\mathbf{u}_{j}(t)=\left(u_{1}^{j}(t) \cdots u_{m}^{j}(t)\right)^{T}$ are, respectively, vectors of $\mathbf{x}(t)$ and $\mathbf{u}(t)$ which are considered 
in $t \in S_{j}$. We mention that $x_{i}^{-k_{1}^{i}+j}\left(t-\tau_{i}\right), 1 \leq i \leq p$, is the $i$ th component of $\left(x_{1}^{-k_{1}^{1}+j}\left(t-\tau_{1}\right) \cdots x_{p}^{-k_{1}^{p}+j}\left(t-\tau_{p}\right)\right)^{T}$ where $\left(t-\tau_{i}\right) \epsilon$ $\left[t_{-k_{1}^{i}+j-1}, t_{-k_{1}^{i}+j}\right]$, and $x_{i}^{k_{2}-j}\left(t_{f}-t\right), 1 \leq i \leq p$, is the $i$ th component of $\left(x_{1}^{k_{2}-j}\left(t_{f}-t\right) \cdots x_{p}^{k_{2}-j}\left(t_{f}-t\right)\right)^{T}$ where $\left(t_{f}-t\right) \epsilon$ $\left[t_{k_{2}-j-1}, t_{k_{2}-j}\right]$. Also

$$
\begin{aligned}
& k_{1}^{i}=\left\{\begin{array}{ll}
\frac{\tau_{i}}{h}, & \frac{\tau_{i}}{h} \in \mathbf{N}, \\
\left(\left[\frac{\tau_{i}}{h}\right]+1\right), & \frac{\tau_{i}}{h} \notin \mathbf{N},
\end{array} \quad 1 \leq i \leq p,\right. \\
& k_{2}= \begin{cases}\frac{t_{f}}{h}, & \frac{t_{f}}{h} \in \mathbf{N}, \\
\left(\left[\frac{t_{f}}{h}\right]+1\right), & \frac{t_{f}}{h} \notin \mathbf{N},\end{cases}
\end{aligned}
$$

where $\left[\tau_{i} / h\right]$ and $\left[t_{f} / h\right]$ denote the integer part of $\tau_{i} / h$ and $t_{f} / h$, respectively.

Our strategy is to use Bézier curves to approximate the solutions $\mathbf{x}_{j}(t)$ and $\mathbf{u}_{j}(t)$ by $\mathbf{v}_{j}(t)$ and $\mathbf{w}_{j}(t)$, respectively, where $\mathbf{v}_{j}(t)$ and $\mathbf{w}_{j}(t)$ are given below. Individual Bézier curves that are defined over the subintervals are joined together to form the Bézier spline curves. For $j=1,2, \ldots, k$, define the Bézier polynomials of degree $n$ that approximate, respectively, the actions of $\mathbf{x}_{j}(t)$ and $\mathbf{u}_{j}(t)$ over the interval $\left[t_{j-1}, t_{j}\right]$ as follows:

$$
\begin{aligned}
& \mathbf{v}_{j}(t)=\sum_{r=0}^{n} \mathbf{a}_{r}^{j} B_{r, n}\left(\frac{t-t_{j-1}}{h}\right), \\
& \mathbf{w}_{j}(t)=\sum_{r=0}^{n} \mathbf{b}_{r}^{j} B_{r, n}\left(\frac{t-t_{j-1}}{h}\right),
\end{aligned}
$$

where

$$
B_{r, n}\left(\frac{t-t_{j-1}}{h}\right)=\left(\begin{array}{l}
n \\
r
\end{array}\right) \frac{1}{h^{n}}\left(t_{j}-t\right)^{n-r}\left(t-t_{j-1}\right)^{r}
$$

is the Bernstein polynomial of degree $n$ over the interval $\left[t_{j-1}, t_{j}\right], \mathbf{a}_{r}^{j}$ and $\mathbf{b}_{r}^{j}$ are, respectively, $p$ and $m$ ordered vectors from the control points (see [22]). By substituting (6) in (4), $R_{1, j}(t)$ for $t \in\left[t_{j-1}, t_{j}\right]$ can be defined as follows:

$$
\begin{aligned}
R_{1, j}(t)= & \dot{\mathbf{v}}_{j}(t)-A(t) \mathbf{v}_{j}(t) \\
& -C(t)\left(v_{1}^{-k_{1}^{1}+j}\left(t-\tau_{1}\right) \cdots v_{p}^{-k_{1}^{p}+j}\left(t-\tau_{p}\right)\right)^{T} \\
& -D(t)\left(v_{1}^{k_{2}-j}\left(t_{f}-t\right) \cdots v_{p}^{k_{2}-j}\left(t_{f}-t\right)\right)^{T} \\
& -G(t) \mathbf{w}_{j}(t) .
\end{aligned}
$$

Let $\mathbf{v}(t)=\sum_{j=1}^{k} \chi_{j}^{1}(t) \mathbf{v}_{j}(t)$ and $\mathbf{w}(t)=\sum_{j=1}^{k} \chi_{j}^{2}(t) \mathbf{w}_{j}(t)$ where $\chi_{j}^{1}(t)$ and $\chi_{j}^{2}(t)$ are, respectively, characteristic function of $\mathbf{v}_{j}(t)$ and $\mathbf{w}_{j}(t)$ for $t \in\left[t_{j-1}, t_{j}\right]$. Beside the boundary conditions on $\mathbf{v}(t)$, at each node, we need to impose the continuity condition on each successive pair of $\mathbf{v}_{j}(t)$ to guarantee the smoothness.
Since the differential equation is of first order, the continuity of $\mathbf{x}$ (or $\mathbf{v}$ ) and its first derivative gives

$$
\mathbf{v}_{j}^{(s)}\left(t_{j}\right)=\mathbf{v}_{j+1}^{(s)}\left(t_{j}\right), \quad s=0,1, j=1,2, \ldots, k-1,
$$

where $\mathbf{v}_{j}^{(s)}\left(t_{j}\right)$ is the $s$ th derivative $\mathbf{v}_{j}(t)$ with respect to $t$ at $t=t_{j}$.

Thus, the vector of control points $\mathbf{a}_{r}^{j}(r=0,1, n-1, n)$ must satisfy (see the Appendix)

$$
\begin{aligned}
\mathbf{a}_{n}^{j}\left(t_{j}-t_{j-1}\right)^{n} & =\mathbf{a}_{0}^{j+1}\left(t_{j+1}-t_{j}\right)^{n}, \\
\left(\mathbf{a}_{n}^{j}-\mathbf{a}_{n-1}^{j}\right)\left(t_{j}-t_{j-1}\right)^{n-1} & =\left(\mathbf{a}_{1}^{j+1}-\mathbf{a}_{0}^{j+1}\right)\left(t_{j+1}-t_{j}\right)^{n-1} .
\end{aligned}
$$

According to the definition of the $t_{i}=t_{0}+i h$ we get that $t_{j}-t_{j-1}=h$. Therefore,

$$
\begin{aligned}
\mathbf{a}_{n}^{j} & =\mathbf{a}_{0}^{j+1}, \\
\left(\mathbf{a}_{n}^{j}-\mathbf{a}_{n-1}^{j}\right) & =\left(\mathbf{a}_{1}^{j+1}-\mathbf{a}_{0}^{j+1}\right) .
\end{aligned}
$$

One may recall that $\mathbf{a}_{r}^{j}$ is a $p$ ordered vector. This approach is called the subdivision scheme (or $h$-refinement in the finite element literature). This method is based on the controlpoint-based method.

Remark 1. By considering the $C^{1}$ continuity of $\mathbf{w}$, the following constraints will be added to constraints in (10):

$$
\begin{aligned}
\mathbf{b}_{n}^{j}\left(t_{j}-t_{j-1}\right)^{n} & =\mathbf{b}_{0}^{j+1}\left(t_{j+1}-t_{j}\right)^{n}, \\
\left(\mathbf{b}_{n}^{j}-\mathbf{b}_{n-1}^{j}\right)\left(t_{j}-t_{j-1}\right)^{n-1} & =\left(\mathbf{b}_{1}^{j+1}-\mathbf{b}_{0}^{j+1}\right)\left(t_{j+1}-t_{j}\right)^{n-1},
\end{aligned}
$$

where the so-called $\mathbf{b}_{r}^{j}(r=0,1, n-1, n)$ is an $m$ ordered vector.

Now, the residual function can be defined in $S_{j}$ as follows:

$$
R_{j}=\int_{t_{j-1}}^{t_{j}}\left\|R_{1, j}(t)\right\|^{2} d t
$$

where $\|\cdot\|$ is the Euclidean norm (recall that $R_{1, j}(t)$ is a $p$ vector where $t \in S_{j}$ ).

Our aim is to solve the following problem over $S=$ $\bigcup_{j=1}^{k} S_{j}$ :

$$
\begin{aligned}
\min & \sum_{j=1}^{k} R_{j} \\
\text { s.t. } & \mathbf{a}_{n}^{j}=\mathbf{a}_{0}^{j+1}, \\
& \left(\mathbf{a}_{n}^{j}-\mathbf{a}_{n-1}^{j}\right)=\left(\mathbf{a}_{1}^{j+1}-\mathbf{a}_{0}^{j+1}\right), \quad j=1,2, \ldots, k-1 .
\end{aligned}
$$

The mathematical programming problem (14) can be solved by many subroutine algorithms. Here, we used Maple 12 to solve this optimization problem. 
Remark 2. Consider the following boundary value problem:

$$
\begin{gathered}
\dot{\mathbf{y}}(t)=R(t) \mathbf{y}(t)+Q(t) \mathbf{y}(t-\alpha)+S(t) \mathbf{z}(t)+\mathbf{a}(t), \\
\dot{\mathbf{z}}(t)=V(t) \mathbf{y}(t)+K(t) \mathbf{z}(t+\alpha)+W(t) \mathbf{z}(t)+\mathbf{b}(t), \\
\mathbf{y}\left(t_{0}\right)=\mathbf{y}_{0}, \\
\mathbf{y}(t)=\boldsymbol{\phi}(t), \quad t \in\left[-\alpha, t_{0}\right), \\
\mathbf{z}\left(t_{f}\right)=\mathbf{z}_{0}, \\
\mathbf{z}(t)=\psi(t), \quad t \in\left(t_{f}, t_{f}+\alpha\right],
\end{gathered}
$$

where $\mathbf{y}(t), \mathbf{z}(t), \mathbf{a}(t), \mathbf{b}(t), \boldsymbol{\phi}(t)$, and $\psi(t)$ are the vectors of appropriate dimensions. $R(t), Q(t), S(t), V(t), K(t)$, and $W(t)$ are the matrices of appropriate dimensions, and $\alpha$ is nonnegative constant time delay.

Let

$$
\mathbf{x}(t)=\left[\begin{array}{ll}
\mathbf{y}(t)^{T} & \mathbf{z}\left(t_{f}-t\right)^{T}
\end{array}\right]^{T}
$$

where $T$ is the transpose; then

$$
\dot{\mathbf{x}}(t)=\left[\dot{\mathbf{y}}^{T}(t)-\dot{\mathbf{z}}^{T}\left(t_{f}-t\right)\right]^{T}
$$

satisfies that

$$
\begin{gathered}
\dot{\mathbf{x}}(t)=A(t) \mathbf{x}(t)+C(t) \mathbf{x}(t-\alpha) \\
+D(t) \mathbf{x}\left(t_{f}-t\right)+\mathbf{u}(t), \quad t \in\left[t_{0}, t_{f}\right], \\
\mathbf{x}\left(t_{0}\right)=\mathbf{x}_{0}=\left[\begin{array}{ll}
\mathbf{y}_{0}^{T} & \mathbf{z}_{0}^{T}
\end{array}\right]^{T},
\end{gathered}
$$

where

$$
\begin{gathered}
A(t)=E_{11}^{(2)} \otimes R(t)-E_{22}^{(2)} \otimes W\left(t_{f}-t\right), \\
C(t)=E_{11}^{(2)} \otimes Q(t)-E_{22}^{(2)} \otimes K\left(t_{f}-t\right), \\
D(t)=E_{12}^{(2)} \otimes S(t)-E_{21}^{(2)} \otimes V\left(t_{f}-t\right), \\
\mathbf{u}(t)=\left[\mathbf{a}^{T}(t)-\mathbf{b}^{T}\left(t_{f}-t\right)\right]^{T},
\end{gathered}
$$

where $E_{i j}^{(f)}$ is the $f \times f$ matrix with 1 at its entry $(i, j)$ and zeros elsewhere and $\otimes$ is Kronecker product (see, e.g., $[4,38,39]$ ).

Remark 3. Now, the following delay differential equation can be considered:

$$
\dot{x}(t)=f(t, x(t), x(t-\tau(t, x(t)))), \quad t \geq 0
$$

with initial condition

$$
x(t)=\phi(t), \quad t \in[-\lambda, 0],
$$

where $\lambda \equiv \inf \{t-\tau(t, u): t \geq 0, u \in \mathbf{R}\}$. In the case when $\lambda$ is not finite, $[-\lambda, 0]$ denotes the interval $(-\infty, 0]$.

Furthermore, we assume that

$$
\tau(t, u) \geq 0, \quad \forall t \geq 0, u \in \mathbf{R}
$$

that is, (20) is a delay differential equation. The existence and uniqueness of the solution of initial value problem (20)-(21) was stated in [40].

Equation (20) is converted into a nonlinear programming problem (NLP) by applying Bézier control points method, whereas the MATLAB optimization routine FMINCON is used for solving resulting NLP. Numerical example shows that the proposed method is efficient and very easy to use.

Remark 4. Now, we limit ourselves to consider the following nonlinear delay differential equation in the type

$$
L x(t)=F(t, x(t), x(\tau(t))), \quad t_{0} \leq t \leq t_{f}
$$

with the following initial conditions:

$$
\begin{gathered}
x^{(k)}\left(t_{0}\right)=x_{0}^{k}, \quad k=0,1, \ldots, n-1, \\
x(t)=\phi(t), \quad t \leq t_{0},
\end{gathered}
$$

where the differential operator $L$ is defined by $L(\cdot)=$ $d^{n}(\cdot) / d t^{n}$.

\section{Convergence Analysis}

In this section, without loss of generality, we analyze the convergence of the control-point-based method applied to the problem (2) with time delay in state when $p=m=1$, and the time interval is $[0,1]$. So, the following problem is considered:

$$
\begin{gathered}
L\left(x(t), u(t), x(t-\tau), x(1-t), \frac{d x(t)}{d t}\right)=\frac{d x(t)}{d t} \\
-A(t) x(t)-C(t) x(t-\tau)-G(t) u(t) \\
-D(t) x(1-t)=F(t), \quad t \in[0,1], \\
x(t)=x_{0}=a, \quad t \leq 0, \quad x(1)=x_{f}=b, \\
u(t)=u_{0}=a_{1}, \quad t \leq 0,
\end{gathered}
$$

where $x(t) \in R, u(t) \in R$, and $a, b, a_{1}$ are given real numbers and $A(t), C(t), G(t), D(t)$, and $F(t)$ are known polynomials for $t \in[0,1]$. The constant time delay $\tau$ is nonnegative.

Without loss of generality, we consider the interval $[0,1]$ instead of $\left[t_{0}, t_{f}\right]$ since the variable $t$ can be changed with the new variable $z$ by $t=\left(t_{f}-t_{0}\right) z+t_{0}$ where $z \in[0,1]$.

Lemma 5. For a polynomial in Bézier form

$$
x(t)=\sum_{i=0}^{n_{1}} a_{i, n_{1}} B_{i, n_{1}}(t),
$$

we have

$$
\begin{aligned}
\frac{\sum_{i=0}^{n_{1}} a_{i, n_{1}}^{2}}{n_{1}+1} & \geq \frac{\sum_{i=0}^{n_{1}+1} a_{i, n_{1}+1}^{2}}{n_{1}+2} \geq \cdots \\
& \geq \frac{\sum_{i=0}^{n_{1}+m_{1}} a_{i, n_{1}+m_{1}}^{2}}{n_{1}+m_{1}+1} \longrightarrow \int_{0}^{1} x^{2}(t) d t, \quad m_{1} \longrightarrow+\infty,
\end{aligned}
$$


where $a_{i, n_{1}+m_{1}}$ is the Bézier coefficient of $x(t)$ after degreeelevating to degree $n_{1}+m_{1}$.

Proof. See [22, page 245].

The convergence of the approximate solution could be done in two ways:

(1) degree raising the Bezier polynomial approximation,

(2) subdivision of the time interval.

In the following, the convergence in each case can be proven, although in numerical examples, we used only subdivision case (see [32]).

\subsection{Degree Raising}

Theorem 6. If the problem (25) with inverse time in state has a unique $C^{1}$ continuous trajectory solution $\bar{x}, C^{0}$ continuous control solution $\bar{u}$, then the approximate solution obtained by the control-point-based method converges to the exact solution $(\bar{x}, \bar{u})$ as the degree of the approximate solution tends to infinity.

Proof. Given an arbitrary small positive number $\epsilon>$ 0 , by the Weierstrass theorem (see [41]), one can easily find polynomials $Q_{1, N_{1}}(t)$ of degree $N_{1}$ and $Q_{2, N_{2}}(t)$ of degree $N_{2}$ such that $\left\|d^{i} Q_{1, N_{1}}(t) / d t^{i}-d^{i} \bar{x}(t) / d t^{i}\right\|_{\infty} \leq \epsilon / 16$, $\left\|d^{i} Q_{1, N_{1}}(t-\tau) / d t^{i}-d^{i} \bar{x}(t-\tau) / d t^{i}\right\|_{\infty} \leq \epsilon / 16, i=0,1$, $\left\|Q_{2, N_{2}}(t)-\bar{u}(t)\right\|_{\infty} \leq \epsilon / 16$, and $\left\|Q_{1, N_{1}}(1-t)-\bar{x}(1-t)\right\|_{\infty} \leq$ $\epsilon / 16$, where $\|\cdot\|_{\infty}$ stands for the $L_{\infty}$-norm over $[0,1]$. Especially, we have

$$
\begin{aligned}
\left\|a-Q_{1, N_{1}}(0)\right\|_{\infty} & \leq \frac{\epsilon}{16}, \\
\left\|b-Q_{1, N_{1}}(1)\right\|_{\infty} & \leq \frac{\epsilon}{16}, \\
\left\|a_{1}-Q_{2, N_{2}}(0)\right\|_{\infty} & \leq \frac{\epsilon}{16} .
\end{aligned}
$$

In general, $Q_{1, N_{1}}(t)$ and $Q_{2, N_{2}}(t)$ do not satisfy the boundary conditions. After a small perturbation with linear and constant polynomials $\alpha t+\beta, \gamma$, respectively, for $Q_{1, N_{1}}(t)$ and $Q_{2, N_{2}}(t)$, we can obtain polynomials $P_{1, N_{1}}(t)=Q_{1, N_{1}}(t)+$ $(\alpha t+\beta)$ and $P_{2, N_{2}}(t)=Q_{2, N_{2}}(t)+\gamma$ such that $P_{1, N_{1}}(t)$ satisfies the boundary conditions $P_{1, N_{1}}(0)=a, P_{1, N_{1}}(1)=b$, and $P_{2, N_{2}}(0)=a_{1}$. Thus, $Q_{1, N_{1}}(0)+\beta=a$, and $Q_{1, N_{1}}(1)+\alpha+\beta=b$. By using (28), one has

$$
\begin{gathered}
\left\|b-Q_{1, N_{1}}(1)\right\|_{\infty}=\|\alpha+\beta\|_{\infty} \leq \frac{\epsilon}{16}, \\
\left\|a-Q_{1, N_{1}}(0)\right\|_{\infty}=\|\beta\|_{\infty} \leq \frac{\epsilon}{16} .
\end{gathered}
$$

Since

$$
\|\alpha\|_{\infty}-\|\beta\|_{\infty} \leq\|\alpha+\beta\|_{\infty} \leq \frac{\epsilon}{16}
$$

so,

$$
\|\alpha\|_{\infty} \leq \frac{\epsilon}{16}+\|\beta\|_{\infty} \leq \frac{\epsilon}{16}+\frac{\epsilon}{16}=\frac{\epsilon}{8} .
$$

By the time, from $a_{1}=P_{2, N_{2}}(0)=Q_{2, N_{2}}(0)+\gamma$,

$$
\left\|a_{1}-Q_{2, N_{2}}(0)\right\|_{\infty}=\|\gamma\|_{\infty} \leq \frac{\epsilon}{16} .
$$

Now, we have

$$
\begin{aligned}
\left\|P_{1, N_{1}}(t)-\bar{x}(t)\right\|_{\infty}= & \left\|Q_{1, N_{1}}(t)+\alpha t+\beta-\bar{x}(t)\right\|_{\infty} \\
\leq & \left\|Q_{1, N_{1}}(t)-\bar{x}(t)\right\|_{\infty} \\
& +\|\alpha+\beta\|_{\infty} \\
\leq & \frac{\epsilon}{8}<\frac{\epsilon}{5}, \\
\left\|\frac{d P_{1, N_{1}}(t)}{d t}-\frac{d \bar{x}(t)}{d t}\right\|_{\infty}= & \left\|\frac{d Q_{1, N_{1}}(t)}{d t}+\alpha-\frac{d \bar{x}(t)}{d t}\right\|_{\infty} \\
\leq & \left\|\frac{d Q_{1, N_{1}}(t)}{d t}-\frac{d \bar{x}(t)}{d t}\right\|_{\infty} \\
& +\|\alpha\|_{\infty} \leq \frac{3 \epsilon}{16}<\frac{\epsilon}{5}, \\
= & \left\|Q_{2, N_{2}}(t)+\gamma-\bar{u}(t)\right\|_{\infty} \\
\leq & \left\|Q_{2, N_{2}}(t)-\bar{u}(t)\right\|_{\infty}+\|\gamma\|_{\infty} \\
\leq & \frac{\epsilon}{8}<\frac{\epsilon}{5},
\end{aligned}
$$

so,

$$
\begin{gathered}
\left\|P_{1, N_{1}}(t-\tau)-\bar{x}(t-\tau)\right\|_{\infty}<\frac{\epsilon}{5}, \\
\left\|\frac{d P_{1, N_{1}}(t-\tau)}{d t}-\frac{d \bar{x}(t-\tau)}{d t}\right\|_{\infty}<\frac{\epsilon}{5}, \\
\left\|P_{1, N_{1}}(1-t)-\bar{x}(1-t)\right\|_{\infty}<\frac{\epsilon}{5} .
\end{gathered}
$$

Now, let $L P_{N}(t)=L\left(P_{1, N_{1}}(t), P_{2, N_{2}}(t), P_{1, N_{1}}(t-\tau), P_{1, N_{1}}(1-t)\right.$, $\left.d P_{1, N_{1}}(t) / d t\right)=d P_{1, N_{1}}(t) / d t-A(t) P_{1, N_{1}}(t)-C(t) P_{1, N_{1}}(t-\tau)-$ $G(t) P_{2, N_{2}}(t)-D(t) P_{1, N_{1}}(1-t)=F(t)$, for every $t \in[0,1]$. Thus, for $N \geq \max \left\{N_{1}, N_{2}\right\}$, an upper bound is found for the following residual:

$$
\begin{aligned}
& \left\|L P_{N}(t)-F(t)\right\|_{\infty} \\
& =\| L\left(P_{1, N_{1}}(t), P_{2, N_{2}}(t), P_{1, N_{1}}(t-\tau),\right. \\
& \left.\quad P_{1, N_{1}}(1-t), \frac{d P_{1, N_{1}}(t)}{d t}\right)-F(t) \|_{\infty}
\end{aligned}
$$




$$
\begin{aligned}
\leq & \left\|\frac{d P_{1, N_{1}}(t)}{d t}-\frac{d \bar{x}(t)}{d t}\right\|_{\infty} \\
& +\|A(t)\|_{\infty}\left\|P_{1, N_{1}}(t)-\bar{x}(t)\right\|_{\infty} \\
& +\|C(t)\|_{\infty}\left\|P_{1, N_{1}}(t-\tau)-\bar{x}(t-\tau)\right\|_{\infty} \\
& +\|G(t)\|_{\infty}\left\|P_{2, N_{2}}(t)-\bar{u}(t)\right\|_{\infty} \\
& +\|D(t)\|_{\infty}\left\|P_{1, N_{1}}(1-t)-\bar{x}(1-t)\right\|_{\infty} \\
\leq & C_{1}\left(\frac{\epsilon}{5}+\frac{\epsilon}{5}+\frac{\epsilon}{5}+\frac{\epsilon}{5}+\frac{\epsilon}{5}\right)=C_{1} \epsilon,
\end{aligned}
$$

where $C_{1}=1+\|A(t)\|_{\infty}+\|C(t)\|_{\infty}+\|G(t)\|_{\infty}+\|D(t)\|_{\infty}$ is a constant.

Since the residual $R\left(P_{N}\right):=L P_{N}(t)-F(t)$ is a polynomial, it can be represented by a Bézier form. Therefore, we have

$$
R\left(P_{N}\right):=\sum_{i=0}^{m_{1}} d_{i, m_{1}} B_{i, m_{1}}(t) .
$$

Then, by Lemma 5, there exists an integer $M(\geq N)$ such that, when $m_{1}>M$, we have

$$
\left|\frac{1}{m_{1}+1} \sum_{i=0}^{m_{1}} d_{i, m_{1}}^{2}-\int_{0}^{1}\left(R\left(P_{N}\right)\right)^{2} d t\right|<\epsilon
$$

which gives

$$
\begin{gathered}
\frac{1}{m_{1}+1} \sum_{i=0}^{m_{1}} d_{i, m_{1}}^{2}<\epsilon+\int_{0}^{1}\left(R\left(P_{N}\right)\right)^{2} d t \\
\leq \epsilon+C_{1}^{2} \epsilon^{2} .
\end{gathered}
$$

Suppose $x(t)$ and $u(t)$ are approximated solution of (25) obtained by the control-point-based method of degree $m_{2}$ $\left(m_{2} \geq m_{1} \geq M\right)$. Let

$$
\begin{aligned}
& R\left(x(t), u(t), x(t-\tau), x(1-t), \frac{d x(t)}{d t}\right) \\
& \quad=L\left(x(t), u(t), x(t-\tau), x(1-t), \frac{d x(t)}{d t}\right)-F(t) \\
& \quad=\sum_{i=0}^{m_{2}} c_{i, m_{2}} B_{i, m_{2}}(t), \quad m_{2} \geq m_{1} \geq M, t \in[0,1] .
\end{aligned}
$$

Define the following norm for difference approximated solution $(x(t), u(t))$ and exact solution $(\bar{x}(t), \bar{u}(t))$ :

$$
\begin{aligned}
& \|(x(t), u(t))-(\bar{x}(t), \bar{u}(t))\| \\
& :=\int_{0}^{1} \sum_{j=0}^{1}\left|\frac{d^{j} x(t)}{d t^{j}}-\frac{d^{j} \bar{x}(t)}{d t^{j}}\right|^{2} d t \\
& \quad+\int_{0}^{1}|u(0)-\bar{u}(0)| d t .
\end{aligned}
$$

By (40), Lemma 5 , the boundary conditions $\bar{x}(0)=a=$ $P_{1, N_{1}}(0)=x(0), \bar{x}(1)=b=P_{1, N_{1}}(1)=\bar{x}(1)$, and $\bar{u}(0)=$ $a_{1}=P_{2, N_{2}}(0)=u(0)$, one can show that

$$
\begin{aligned}
& \|(x(t), u(t))-(\bar{x}(t), \bar{u}(t))\| \\
& \leq C(|x(0)-\bar{x}(0)| \\
& +|x(1)-\bar{x}(1)|+|u(0)-\bar{u}(0)| \\
& +\| R\left(\left(x(t), u(t), x(t-\tau), x(1-t), \frac{d x(t)}{d t}\right)\right. \\
& -(\bar{x}(t), \bar{u}(t), \bar{x}(t-\tau), \bar{x}(1-t), \\
& \left.\left.\left.\frac{d \bar{x}(t)}{d t}\right)\right) \|_{2}^{2}\right) \\
& =C \int_{0}^{1} \sum_{i=0}^{m_{2}}\left(c_{i, m_{2}} B_{i, m_{2}}(t)\right)^{2} d t \\
& \leq \frac{C}{m_{2}+1} \sum_{i=0}^{m_{2}} c_{i, m_{2}}^{2} \text {. }
\end{aligned}
$$

The last inequality in (41) is obtained by Lemma 5 , where $C$ is a constant positive number. Now

$$
\begin{aligned}
\|(x(t), u(t))-(\bar{x}(t), \bar{u}(t))\| & \leq \frac{C}{m_{2}+1} \sum_{i=0}^{m_{2}} c_{i, m_{2}}^{2} \\
& \leq \frac{C}{m_{2}+1} \sum_{i=0}^{m_{2}} d_{i, m_{2}}^{2} \\
& \leq \frac{C}{m_{1}+1} \sum_{i=0}^{m_{1}} d_{i, m_{1}}^{2} \\
& \leq C\left(\epsilon+C_{1}^{2} \epsilon^{2}\right) \\
& =\epsilon_{1}, \quad m_{1} \geq M,
\end{aligned}
$$

where the last inequality in (42) comes from (36). This completes the proof.

\subsection{Subdivision}

Theorem 7. Let $(x, u)$ be the approximate solution of the problem (25) with inverse time obtained by the subdivision scheme of the control-point-based method. If (25) has a unique solution $(\bar{x}, \bar{u})$ and $(\bar{x}, \bar{u})$ is smooth enough so that the cubic spline $T(\bar{x}, \bar{u})$ interpolates to $(\bar{x}, \bar{u})$ and converges to $(\bar{x}, \bar{u})$ in the order $O\left(h^{q}\right),(q>2)$, where $h$ is the maximal width of all subintervals, then $(x, u)$ converges to $(\bar{x}, \bar{u})$ as $h \rightarrow 0$.

Proof. We first impose a uniform partition $\prod_{d}=\bigcup_{i}\left[t_{i}, t_{i+1}\right]$ on the interval $[0,1]$ as $t_{i}=i d$, where $d=1 /\left(n_{1}+1\right)$. 
Let $I_{d}(\bar{x}(t), \bar{u}(t), \bar{x}(t-\tau), \bar{x}(1-t), d \bar{x}(t) / d t)$ be the cubic spline over $\prod_{d}$ which is interpolating to $(\bar{x}, \bar{u})$. Then, for an arbitrary small positive number $\epsilon>0$, there exists a $\delta_{1}>0$ such that

$$
\begin{aligned}
& \| L\left(\bar{x}(t), \bar{u}(t), \bar{x}(t-\tau), \bar{x}(1-t), \frac{d \bar{x}(t)}{d t}\right) \\
& -L\left(I_{d}\left(\bar{x}(t), \bar{u}(t), \bar{x}(t-\tau), \bar{x}(1-t), \frac{d \bar{x}(t)}{d t}\right)\right) \|_{\infty} \leq \epsilon
\end{aligned}
$$

provided that $d<\delta_{1}$. Let $R\left(I_{d}(\bar{x}(t), \bar{u}(t), \bar{x}(t \quad-\right.$ $\tau), \bar{x}(1-t), d \bar{x}(t) / d t))=L\left(I_{d}(\bar{x}(t), \bar{u}(t), \bar{x}(t-\tau), \bar{x}(1-\right.$ $t), d \bar{x}(t) / d t))-F(t)$ be the residual. For each subinterval $\left[t_{i}, t_{i+1}\right], \quad R\left(I_{d}(\bar{x}(t), \bar{u}(t), \bar{x}(t-\tau), \bar{x}(1-t), d \bar{x}(t) / d t)\right)$ is a polynomial. On each interval $\left[t_{i}, t_{i+1}\right]$, we impose another uniform partition $\prod_{i, h}=\bigcup_{j}\left[t_{i, j}, t_{i, j+1}\right]$ as $t_{i, j}=i d+j h$ where $h=d /\left(m_{1}+1\right), j=0, \ldots, m_{1}$. Express $R\left(I_{d}(\bar{x}(t), \bar{u}(t), \bar{x}(t-\tau), \bar{x}(1-t), d \bar{x}(t) / d t)\right)$ in $\left[t_{i, j-1}, t_{i, j}\right]$ as

$$
\begin{gathered}
R\left(I_{d}\left(\bar{x}(t), \bar{u}(t), \bar{x}(t-\tau), \bar{x}(1-t), \frac{d \bar{x}(t)}{d t}\right)\right) \\
=\sum_{p_{1}=0}^{l} r_{p_{1}}^{i, j} B_{p_{1}, l}(t), \quad t \in\left[t_{i, j-1}, t_{i, j}\right] .
\end{gathered}
$$

By Lemma 3 in [22], there exists a $\delta_{2}>0\left(\delta_{2} \leq \delta_{1}\right)$ such that, when $h<\delta_{2}$, we have

$$
\begin{aligned}
\mid \sum_{j=1}^{m_{1}}\left(t_{i, j}-t_{i, j-1}\right) \sum_{p_{1}=0}^{l}\left(r_{p_{1}}^{i, j}\right)^{2}-(l+1) \\
\times \int_{t_{i}}^{t_{i+1}} R^{2}\left(I_{d}(\bar{x}(t), \bar{u}(t), \bar{x}(t-\tau),\right. \\
\left.\left.\bar{x}(1-t), \frac{d \bar{x}(t)}{d t}\right)\right) \mid d t \leq \frac{\epsilon}{d} .
\end{aligned}
$$

Thus,

$$
\begin{array}{r}
\mid \sum_{i=1}^{n_{1}} \sum_{j=1}^{m_{1}}\left(t_{i, j}-t_{i, j-1}\right) \sum_{p_{1}=0}^{l}\left(r_{p_{1}}^{i, j}\right)^{2} \\
-(l+1) \int_{0}^{1} R^{2}\left(I_{d}(\bar{x}(t), \bar{u}(t), \bar{x}(t-\tau),\right. \\
\left.\left.\bar{x}(1-t), \frac{d \bar{x}(t)}{d t}\right)\right) \mid d t
\end{array}
$$

or

$$
\begin{aligned}
& \sum_{i=1}^{n_{1}} \sum_{j=1}^{m_{1}}\left(t_{i, j}-t_{i, j-1}\right) \sum_{p_{1}=0}^{l}\left(r_{p_{1}}^{i, j}\right)^{2} \\
& <(l+1) \int_{0}^{1} R^{2}\left(I _ { d } \left(\frac{d \bar{x}(t)}{d t} \bar{x}(t), \bar{u}(t), \bar{x}(t-\tau),\right.\right. \\
& \left.\left.\bar{x}(1-t), \frac{d \bar{x}(t)}{d t}\right)\right) d t+\epsilon \\
& <(l+1) \epsilon^{2}+\epsilon .
\end{aligned}
$$

Now combining the partitions $\prod_{d}$ and all $\prod_{i, h}$ gives a denser partition with the length $h$ for each subinterval. Suppose $(x(t), u(t))$ is the approximate solution by the control-pointbased method with respect to this partition, and denote the residual over $\left[t_{i, j-1}, t_{i, j}\right]$ by

$$
\begin{aligned}
R & \left(x(t), u(t), x(t-\tau), x(1-t), \frac{d x(t)}{d t}\right) \\
& =L\left(x(t), u(t), x(t-\tau), x(1-t), \frac{d x(t)}{d t}\right)-F(t) \\
& =\sum_{p_{1}=0}^{l} c_{p_{1}}^{i, j} B_{p_{1}, l}(t) .
\end{aligned}
$$

Define the following norm for difference approximate solution $(x(t), u(t))$ and exact solution $(\bar{x}(t), \bar{u}(t))$ :

$$
\begin{aligned}
& \|(x(t), u(t))-(\bar{x}(t), \bar{u}(t))\| \\
& :=\sum_{i=1}^{n_{1}} \sum_{j=1}^{m_{1}} \int_{t_{i, j-1}}^{t_{i, j}}|x(t)-\bar{x}(t)|^{2} d t \\
& \quad+\sum_{i=1}^{n_{1}} \sum_{j=1}^{m_{1}} \int_{t_{i, j-1}}^{t_{i, j}}\left|\frac{d x(t)}{d t}-\frac{d \bar{x}(t)}{d t}\right|^{2} d t \\
& \quad+\sum_{i=1}^{n_{1}} \sum_{j=1}^{m_{1}} \int_{t_{i, j-1}}^{t_{i, j}}|u(0)-\bar{u}(0)| d t .
\end{aligned}
$$

Then, there is a constant $C$ such that

$$
\begin{aligned}
& \|(x(t), u(t))-(\bar{x}(t), \bar{u}(t))\| \\
& \leq C \| R\left(\left(x(t), u(t), x(t-\tau), x(1-t), \frac{d x(t)}{d t}\right)\right. \\
& \left.\quad-\left(\bar{x}(t), \bar{u}(t), \bar{x}(t-\tau), \bar{x}(1-t), \frac{d \bar{x}(t)}{d t}\right)\right) \|_{2} \\
& \leq \frac{C}{l+1} \sum_{i=1}^{n_{1}} \sum_{j=1}^{m_{1}}\left(t_{i, j}-t_{i, j-1}\right) \sum_{p_{1}=0}^{l}\left(c_{p_{1}}^{i, j}\right)^{2} ;
\end{aligned}
$$


the last inequality in (50) is obtained by Lemma 5 . It can be shown that

$$
\begin{aligned}
& \frac{C}{l+1} \sum_{i=1}^{n_{1}} \sum_{j=1}^{m_{1}}\left(t_{i, j}-t_{i, j-1}\right) \sum_{p_{1}=0}^{l}\left(c_{p_{1}}^{i, j}\right)^{2} \\
& \quad \leq \frac{C}{l+1} \sum_{i=1}^{n_{1}} \sum_{j=1}^{m_{1}}\left(t_{i, j}-t_{i, j-1}\right) \sum_{p_{1}=0}^{l}\left(r_{p_{1}}^{i, j}\right)^{2} \\
& \quad \leq C\left(\epsilon^{2}+\frac{\epsilon}{l+1}\right)=\epsilon_{2} .
\end{aligned}
$$

By Lemma 3 in [22], we conclude that the approximate solution converges to the exact solution in order $o\left(h^{q}\right),(q>$ 2). This completes the proof.

\section{Numerical Examples}

Applying the presented method, in Examples 1, 2, and 3, the Bézier curves are chosen as piecewise polynomials of degree 3.
Example 8. Consider the delay system containing inverse time described by (see [4])

$$
\begin{aligned}
\dot{\mathbf{x}}(t)= & {\left[\begin{array}{cc}
t^{2}+1 & -t^{2} \\
0 & -9
\end{array}\right] \mathbf{x}(t)+\left[\begin{array}{cc}
1 & -1 \\
9 & 0
\end{array}\right] \mathbf{x}\left(t-\frac{1}{3}\right) } \\
& +\left[\begin{array}{cc}
1 & 0 \\
-1 & 1
\end{array}\right] \mathbf{x}(1-t)+\left[\begin{array}{c}
4 t+3 \\
8 t+15
\end{array}\right] u(t), \\
& \phi(t)=\left[\begin{array}{l}
t^{2}-1 \\
t^{2}+1
\end{array}\right], \quad t \in\left[-\frac{1}{3}, 0\right],
\end{aligned}
$$

where we have the following exact solution:

$$
\mathbf{x}(t)=\left[\begin{array}{ll}
x_{1}(t) & x_{2}(t)
\end{array}\right]^{T}=\left[\begin{array}{ll}
t^{2}-1 & t^{2}+1
\end{array}\right]^{T} .
$$

Let $u(t)=1$. Then, by (14) and choosing $n=3, k=6$ we have the approximate solution $\mathbf{x}(t)=\left[\begin{array}{ll}x_{1}(t) & x_{2}(t)\end{array}\right]^{T}$

$$
\begin{aligned}
& x_{1}(t)= \begin{cases}-1.000000001+8.333333337 \times 10^{-9} t+0.9999999669 t^{2}+10^{-7} t^{3}, & 0 \leq t \leq \frac{1}{6}, \\
-0.9999999988+8.13333333 \times 10^{-9} t+0.9999999829 t^{2}, & \frac{1}{6} \leq t \leq \frac{1}{3}, \\
-0.9999999997+2.00 \times 10^{-10} t+t^{2}, & \frac{1}{3} \leq t \leq \frac{1}{2}, \\
-0.9999999927-2.202222223 \times 10^{-8} t+1.000000017 t^{2}, & \frac{1}{2} \leq t \leq \frac{2}{3}, \\
-0.9999999902-1.504444443 \times 10^{-8} t+0.9999999963 t^{2}+10^{-8} t^{3}, & \frac{2}{3} \leq t \leq \frac{5}{6}, \\
-1.000000032+1.120666667 \times 10^{-7} t+0.9999998702 t^{2}+5 \times 10^{-8} t^{3}, & \frac{5}{6} \leq t \leq 1,\end{cases} \\
& x_{2}(t)= \begin{cases}1.000000001+0.000011825 t+0.9996447669 t^{2}+0.0023693 t^{3}, & 0 \leq t \leq \frac{1}{6}, \\
1.000000001+0.00001180813339 t+0.9996447663 t^{2}+0.0023695 t^{3}, & \frac{1}{6} \leq t \leq \frac{1}{3}, \\
0.9999999645+0.00001211131104 t+0.9996439669 t^{2}+0.0023702 t^{3}, & \frac{1}{3} \leq t \leq \frac{1}{2}, \\
1.000000063+0.00001151408882 t+0.9996452169 t^{2}+0.0023693 t^{3}, & \frac{1}{2} \leq t \leq \frac{2}{3}, \\
0.9581187057+0.1594325022 t+0.8040813829 t^{2}+0.0783674 t^{3}, & \frac{2}{3} \leq t \leq \frac{5}{6}, \\
0.9581181451+0.1594344559 t+0.8040791002 t^{2}+0.0783683 t^{3}, & \frac{5}{6} \leq t \leq 1 .\end{cases}
\end{aligned}
$$


The graphs of approximate trajectories are shown in Figures 1 and 2 .

Example 9. Consider the boundary value problem described by (see [4])

$$
\begin{gathered}
\dot{y}(t)=16 t y\left(t-\frac{1}{4}\right)-16 z(t)+8 t^{2}+17 t+16 \\
\dot{z}(t)=64 t y(t)-64 z\left(t+\frac{1}{4}\right)+51 t^{2}+76 t+65 \\
y(t)=t^{2}-1, \quad-\frac{1}{4} \leq t \leq 0 \\
z(t)=t^{3}+1, \quad 1 \leq t \leq \frac{5}{4}
\end{gathered}
$$

From (18), we have (see [4])

$$
\begin{aligned}
\dot{\mathbf{x}}(t)= & {\left[\begin{array}{cc}
16 t & 0 \\
0 & 64
\end{array}\right] \mathbf{x}\left(t-\frac{1}{4}\right)+\left[\begin{array}{cc}
0 & -16 \\
64 t-64 & 0
\end{array}\right] \mathbf{x}(1-t) } \\
& +\left[\begin{array}{c}
8 t^{2}+17 t+16 \\
-51 t^{2}+178 t-62
\end{array}\right], \\
& \phi(t)=\left[\begin{array}{c}
t^{2}-1 \\
-t^{3}+3 t^{2}-3 t+1
\end{array}\right], \quad t \in\left[-\frac{1}{4}, 0\right],
\end{aligned}
$$

where $\mathbf{x}(t)=\left[\begin{array}{ll}x_{1}(t) & x_{2}(t)\end{array}\right]^{T}=\left[\begin{array}{ll}y(t) & z(1-t)\end{array}\right]^{T}$, and we have the following exact solution:

$$
\mathbf{x}(t)=\left[\begin{array}{ll}
x_{1}(t) & x_{2}(t)
\end{array}\right]^{T}=\left[\begin{array}{ll}
t^{2}-1 & t^{3}+1
\end{array}\right]^{T} .
$$

Let $u(t)=1$. Then, by (14) and choosing $n=3, k=4$ we have the approximate solution $\mathbf{x}(t)=\left[\begin{array}{ll}x_{1}(t) & x_{2}(t)\end{array}\right]^{T}$ :

$$
x_{1}(t)= \begin{cases}t^{2}-1, & 0 \leq t \leq \frac{1}{4}, \\ t^{2}-1, & \frac{1}{4} \leq t \leq \frac{1}{2}, \\ t^{2}-1, & \frac{1}{2} \leq t \leq \frac{3}{4}, \\ -1.000000006+2.0625 \times 10^{-8} t & \\ +0.999999975 t^{2}+10^{-8} t^{3}, & \frac{3}{4} \leq t \leq 1,\end{cases}
$$

$$
x_{2}(t)=t^{3}+1
$$

The graphs of approximate trajectories are shown in Figures 3 and 4 .

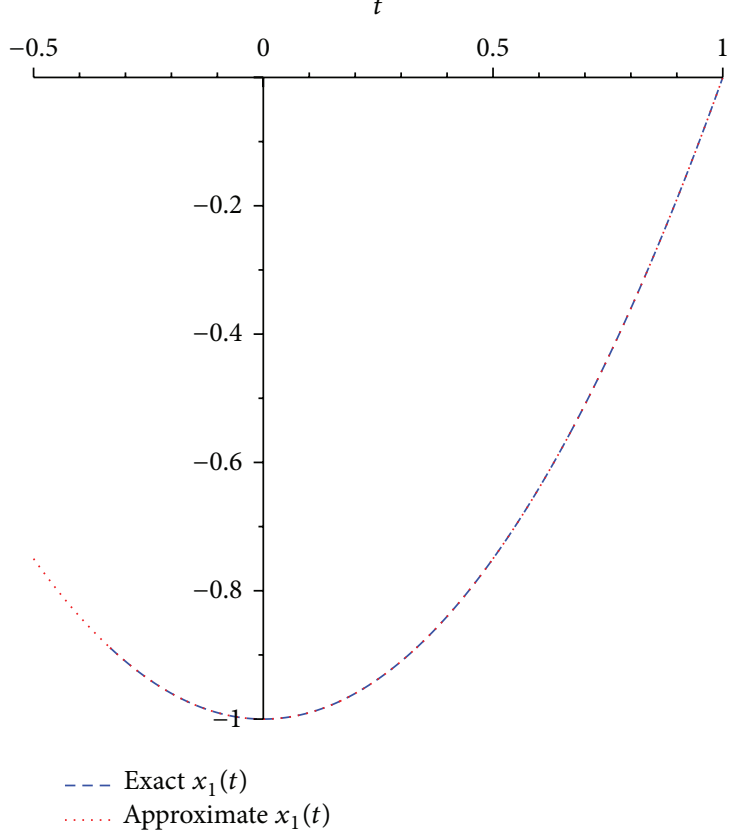

FIGURE 1: The graph of approximated trajectory $x_{1}(t)$ for Example 1 .

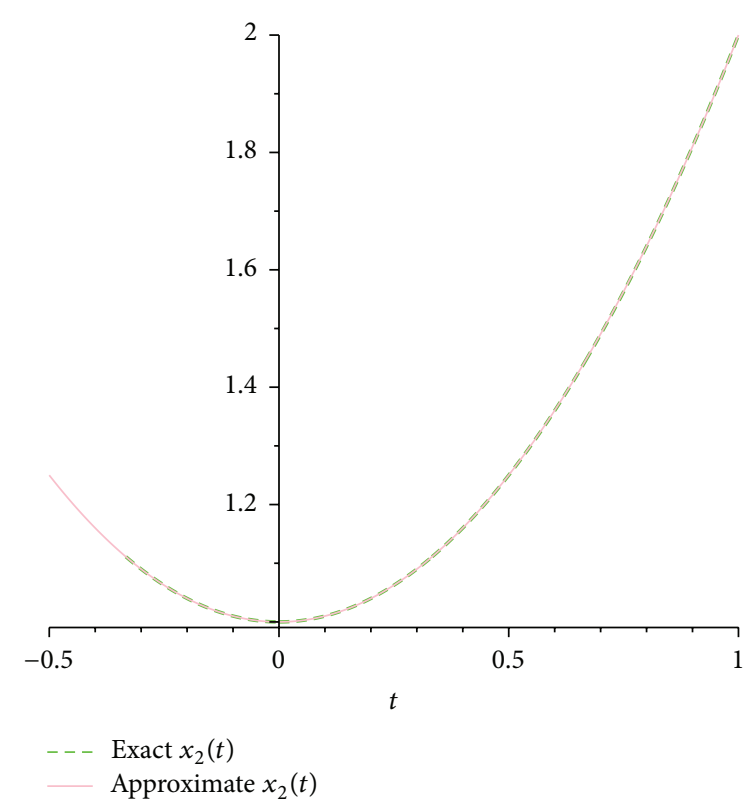

FIgURE 2: The graph of approximated trajectory $x_{2}(t)$ for Example 1.

Example 10. Consider the time-varying delay system described by (see [42])

$$
\begin{gathered}
{\left[\begin{array}{c}
\dot{x}_{1}(t) \\
\dot{x}_{2}(t)
\end{array}\right]=\left[\begin{array}{cc}
0 & 1 \\
-25 & -5 t
\end{array}\right]\left[\begin{array}{l}
x_{1}\left(t-\frac{1}{4}\right) \\
x_{2}\left(t-\frac{1}{4}\right)
\end{array}\right]+\left[\begin{array}{l}
0 \\
1
\end{array}\right],} \\
{\left[\begin{array}{l}
x_{1}(t) \\
x_{2}(t)
\end{array}\right]=\left[\begin{array}{l}
0 \\
0
\end{array}\right], \quad t \in\left[-\frac{1}{4}, 0\right] .}
\end{gathered}
$$


The exact solutions are [42]

$$
\begin{aligned}
& x_{1}(t)=\left\{\begin{array}{lr}
0, & t \in\left[0, \frac{1}{4}\right], \\
\frac{1}{32}-\frac{1}{4} t+\frac{1}{2} t^{2}, & t \in\left[\frac{1}{4}, \frac{1}{2}\right], \\
\frac{1}{32}-\frac{19}{96} t+\frac{3}{16} t^{2}+\frac{5}{8} t^{3}-\frac{5}{12} t^{4}, & t \in\left[\frac{1}{2}, \frac{3}{4}\right], \\
-\frac{9641}{32768}+\frac{37391}{24576} t-\frac{3183}{1024} t^{2}+\frac{7065}{2304} t^{3}-\frac{135}{384} t^{4}-\frac{85}{96} t^{5}+\frac{5}{18} t^{6}, & t \in\left[\frac{3}{4}, 1\right],
\end{array}\right. \\
& x_{2}(t)= \begin{cases}t, & t \in\left[0, \frac{1}{4}\right], \\
-\frac{5}{384}+t+\frac{5}{8} t^{2}-\frac{5}{3} t^{3}, & t \in\left[\frac{1}{4}, \frac{1}{2}\right], \\
\frac{775}{1536}-\frac{17}{8} t+\frac{1295}{192} t^{2}-\frac{115}{24} t^{3}-\frac{75}{32} t^{4}+\frac{5}{3} t^{5}, & t \in\left[\frac{1}{2}, \frac{3}{4}\right], \\
\frac{87997}{132120}-\frac{1051}{1024} t-\frac{95755}{49152} t^{2}+\frac{21515}{1536} t^{3}-\frac{55325}{3072} t^{4}+\frac{335}{96} t^{5}+\frac{2125}{576} t^{6}-\frac{25}{21} t^{7}, & t \in\left[\frac{3}{4}, 1\right] .\end{cases}
\end{aligned}
$$

Here, this problem is solved by choosing $k=8$ and $n=3$. the following approximate solutions $x_{1}(t)$ and $x_{2}(t)$ are found. In
Tables 1 and 2, exact, numerical results of this method and obtained results in [42] are shown, respectively:

$$
x_{1}(t)= \begin{cases}-0.001524977445 t+0.04981148910 t^{2}-0.3456171465 t^{3}, & t \in\left[0, \frac{1}{8}\right], \\ -0.002668294207+0.06251408351 t-0.4625009986 t^{2}+1.020549487 t^{3}, & t \in\left[\frac{1}{8}, \frac{1}{4}\right], \\ 0.006613889339-0.04887212012 t-0.01695618114 t^{2}+0.4264897281 t^{3}, & t \in\left[\frac{1}{4}, \frac{3}{8}\right], \\ 0.01307452454-0.1005572014 t+0.1208707015 t^{2}+0.303976944 t^{3}, & t \in\left[\frac{3}{8}, \frac{1}{2}\right], \\ 0.1271590458-0.7850643303 t+1.489884961 t^{2}-0.608699230 t^{3}, & t \in\left[\frac{1}{2}, \frac{5}{8}\right], \\ 0.06579667219-0.4905249419 t+1.018621948 t^{2}-0.357358960 t^{3}, & t \in\left[\frac{5}{8}, \frac{3}{4}\right], \\ 0.6384881122-2.601997790 t+3.629012898 t^{2}-1.439473220 t^{3}, & t \in\left[\frac{3}{4}, \frac{7}{8}\right], \\ & t \in\left[\frac{7}{8}, 1\right],\end{cases}
$$




$$
x_{2}(t)= \begin{cases}1.003041110 t-0.09123330000 t^{2}+0.6082219700 t^{3}, & t \in\left[0, \frac{1}{8}\right], \\ 0.003925049727+0.9088399072 t+0.6623763650 t^{2}-1.401403820 t^{3}, & t \in\left[\frac{1}{8}, \frac{1}{4}\right], \\ 0.003925049727+0.9088399072 t+0.6623763650 t^{2}-1.401403820 t^{3}, & t \in\left[\frac{1}{4}, \frac{3}{8}\right], \\ -0.02462216250+1.075221794 t+0.4666746125 t^{2}-1.558091100 t^{3}, & t \in\left[\frac{3}{8}, \frac{1}{2}\right], \\ 0.3991598156-1.467470069 t+5.552058325 t^{2}-4.948346900 t^{3}, & t \in\left[\frac{1}{2}, \frac{5}{8}\right], \\ 0.00006281562500+0.4481955219 t+2.486993388 t^{2}-3.313645600 t^{3}, & t \in\left[\frac{5}{8}, \frac{3}{4}\right], \\ -1.159405308+5.086068009 t-3.696836582 t^{2}-0.5652767300 t^{3}, & t \in\left[\frac{3}{4}, \frac{7}{8}\right], \\ -5.634050302+20.42770799 t-21.23013942 t^{2}+6.114076730 t^{3}, & t \in\left[\frac{7}{8}, 1\right] .\end{cases}
$$

Example 11. Consider the following system described by (see [40])

$$
\begin{gathered}
\dot{x}(t)=\frac{8}{t+1} x\left(t-\left(\frac{t}{2}+\frac{1}{2}\right)\right), \quad t \geq 0, \\
x(t)=(t+1)^{2}, \quad t \in\left[-\frac{1}{2}, 0\right] .
\end{gathered}
$$

Analytic solution of the initial value problem (IVP) is $x(t)=$ $(t+1)^{2}$. By choosing $k=1$ and $n=16$ (degree raising), we obtain the following solution:

$$
\begin{aligned}
x(t)= & +0.2018032795 \times 10^{-4} t^{12} \\
& -0.01572515756 t^{7}-0.008572702573 t^{5} \\
& +0.01741959010 t^{6}-0.0001540665901 t^{11} \\
& -0.1834453040 \times 10^{-5} t^{13} \\
& +1.101285958 \times 10^{-7} t^{14}+0.008669328894 t^{8} \\
& +1.999552507 t \\
& +6.306939519 \times 10^{-11} t^{16}-3.928281389 \\
& \times 10^{-9} t^{15}-0.003213347229 t^{9} \\
& +0.9993525856 t^{2}+0.0008342736689 t^{10} \\
& +0.004438985657 t^{3}-0.002620448442 t^{4} .
\end{aligned}
$$

In Table 3, exact and presented methods are shown, respectively.
Example 12. Consider the following system described by (see [40])

$$
\begin{gathered}
\dot{x}(t)=x\left(t-1-\frac{1}{t+1}\right), \quad t \geq 0, \\
x(t)= \begin{cases}\frac{2}{3}(t+2), & -2 \leq t \leq-0.5, \\
1, & -0.5 \leq t \leq 0,\end{cases}
\end{gathered}
$$

where the exact solution is $x(t)=1+(2 / 3) t+t^{3} / 3-$ $(2 / 3) \log (t+1)$ on $[0,1]$ and $x(t)=1-(2 / 3) \log 2+t$ on $[1,2]$. By choosing $k=1$ and $n=7$ (degree raising), we obtain the following solution:

$$
\begin{aligned}
x(t)= & 1+5.424427795 t^{5}-1.611981446 t^{6} \\
& -2.552250886 t^{2}+7.963903747 t^{3} \\
& +0.3574277875 t-9.236517482 t^{4} \\
& +0.1928923646 t^{7} .
\end{aligned}
$$

In Table 4, exact, numerical results of this method, method in [40], error of presented method, and error of the method in [40] are shown, respectively.

Example 13. Consider the following system described by (see [40])

$$
\begin{aligned}
& \dot{x}(t)=-x(t-\tau(t)), \quad t \in[0,2], \\
& x(0)=1 \text {, } \\
& \tau(t) \equiv \begin{cases}t-2+\sqrt{4-2 t}, & 0 \leq t \leq 2, \\
0, & t>2 .\end{cases}
\end{aligned}
$$


The solution of this problem is

$$
x(t)= \begin{cases}\frac{(t-2)^{2}}{4}, & 0 \leq t \leq 2 \\ 0, & t>2\end{cases}
$$

By choosing $k=1$ and $n=7$ (degree raising), we obtain the following solution:

$$
\begin{aligned}
x(t)= & 1-1.000000002 t+3.207267830 \times 10^{-9} t^{6} \\
& +0.2500000112 \times t^{2}-3.416339151 \times 10^{-10} t^{7} \\
& -1.204800000 \times 10^{-8} t^{5}-2.304000000 \times 10^{-8} t^{3} \\
& +2.296000000 \times 10^{-8} t^{4}
\end{aligned}
$$

In Table 5, exact, numerical results of this method, method in [40], error of presented method, and error of the method in [40] are shown, respectively.

Example 14. Consider the following LDDE described by

$$
\frac{d^{3} x(t)}{d t^{3}}=-x(t)-x(t-0.3)+e^{-t+0.3}, \quad 0 \leq t \leq 1
$$

with the initial conditions

$$
\begin{aligned}
& x(0)=1, \quad \frac{d x(0)}{d t}=-1, \quad \frac{d^{2} x(0)}{d t^{2}}=1, \quad x(t)=e^{-t}, \\
& t \leq 0 \text {, }
\end{aligned}
$$

where the exact solution of this example is $x(t)=e^{-t}$. Here, this problem is solved by choosing $k=10$ and $n=3$. The graph of error is shown in Figure 5, and the following approximate solution $x(t)$ is found:

$$
x(t)= \begin{cases}1-t+0.5 t^{2}-0.172928 t^{3}, & t \in[0,0.1], \\ 0.9999767558-0.999302674 t+0.49302674 t^{2}-0.1496838 t^{3}, & t \in[0.1,0.2], \\ 0.9998081522-0.996773576 t+0.48038104 t^{2}-0.1286073 t^{3}, & t \in[0.2,0.3], \\ 0.9992953871-0.991645877 t+0.46328853 t^{2}-0.1096154 t^{3}, & t \in[0.3,0.4], \\ 0.9982244623-0.983613861 t+0.44320829 t^{2}-0.0928817 t^{3}, & t \in[0.4,0.5], \\ 0.9964070488-0.972709334 t+0.42139914 t^{2}-0.0783422 t^{3}, & t \in[0.5,0.6], \\ 0.9937493164-0.9594206 t+0.39925114 t^{2}-0.0660377 t^{3}, & t \in[0.6,0.7], \\ 0.9903114379-0.944686777 t+0.37820273 t^{2}-0.0560146 t^{3}, & t \in[0.7,0.8], \\ 0.9863822587-0.929952279 t+0.35978451 t^{2}-0.0483403 t^{3}, & t \in[0.8,0.9], \\ 0.9825547252-0.917193744 t+0.34560826 t^{2}-0.0430898 t^{3}, & t \in[0.9,1] .\end{cases}
$$

Example 15. Consider the second-order linear decay differential equation:

$$
\begin{gathered}
\ddot{x}(t)=\frac{3}{4} x(t)+x\left(\frac{t}{2}\right)-t^{2}+2, \quad 0 \leq t \leq 1, \\
x(0)=0, \quad \dot{x}(0)=0 .
\end{gathered}
$$

The exact solution of this problem is $x(t)=t^{2}$. Here, this problem is solved by choosing $k=1$ and $n=7$. The following approximate solution $x(t)$ is found.

$$
\begin{aligned}
x(t)= & 1.882848000 t^{2}-5.072623999 t^{3}+15.56400000 t^{4} \\
& -28.14240000 t^{5}+30.84000000 t^{6}-t^{9}+7 t^{8} \\
& -0.06182400000 t \\
& -20.01000000 t^{7} .
\end{aligned}
$$

In Table 6, exact, numerical results of this method, error of presented method, and error of the method in [43] are shown, respectively.

\section{Conclusions}

Using the Bézier curves, the general algorithm is provided for the delay systems containing inverse time. Numerical examples show that the proposed method is efficient and very easy to use.

\section{Appendix}

In this Appendix, we specify the derivative of Bézier curve.

By (6), we have

$$
\mathbf{v}_{j}(t)=\sum_{i=0}^{n} a_{i}^{j} B_{i, n}(t), \quad t \in[0,1],
$$

where $B_{i, n}(t)=(n ! / i !(n-i) !) t^{i}(1-t)^{n-i}$.

Now, we have (see [44])

$$
\frac{d B_{i, n}(t)}{d t}=n\left(B_{i-1, n-1}(t)-B_{i, n-1}(t)\right), \quad 0 \leq i \leq n,
$$




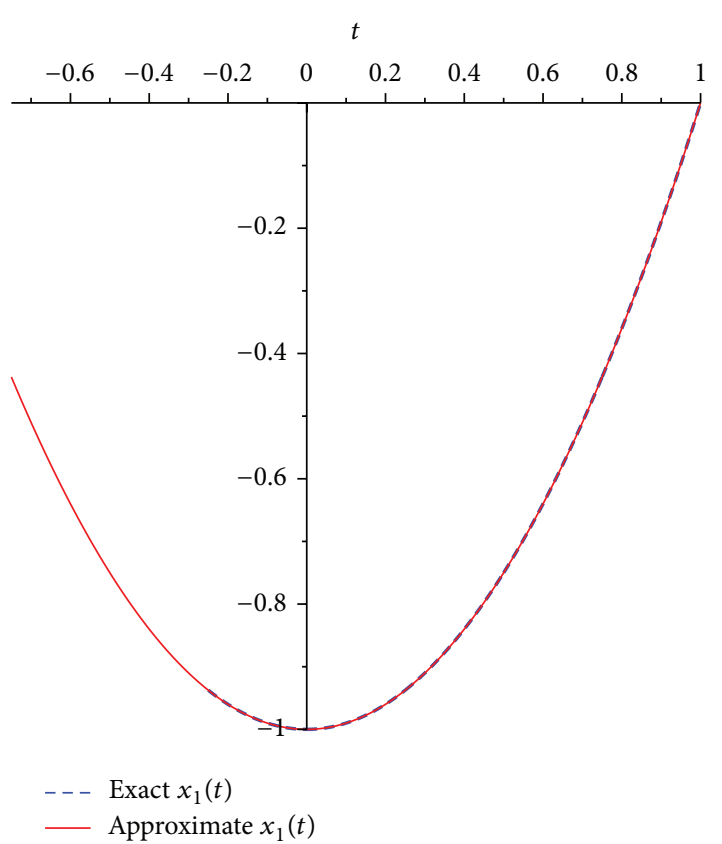

Figure 3: The graph of approximated trajectory $x_{1}(t)$ for Example 2.

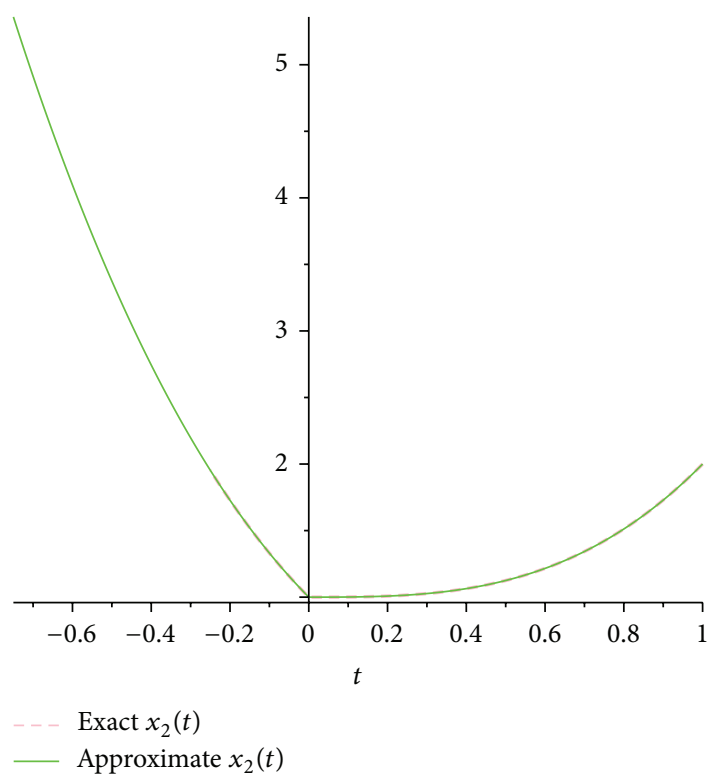

FIGURE 4: The graph of approximated trajectory $x_{2}(t)$ for Example 2.

where $B_{-1, n-1}(t)=B_{n, n-1}(t)=0$, and

$$
\begin{aligned}
B_{i-1, n-1}(t) & =\frac{(n-1) !}{(i-1) !(n-i) !} t^{i-1}(1-t)^{n-i}, \\
B_{i, n-1}(t) & =\frac{(n-1) !}{i !(n-i-1) !} t^{i}(1-t)^{n-i-1} .
\end{aligned}
$$

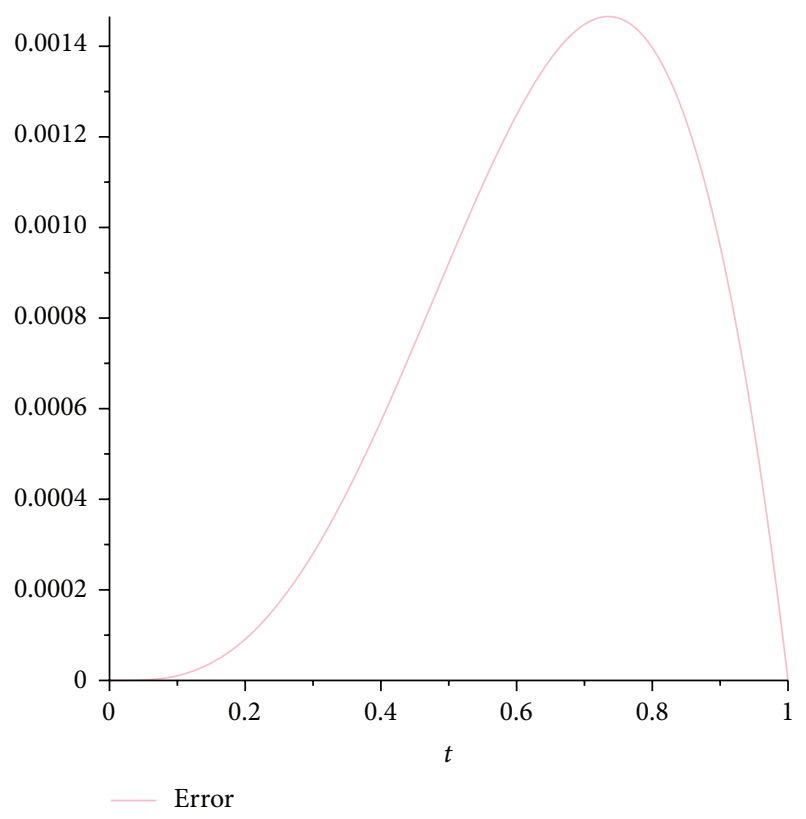

FIgURE 5: The graph of error for Example 7.

By using (A.2), the first derivative $\mathbf{v}_{j}(t)$ is shown as

$$
\begin{aligned}
\frac{d \mathbf{v}_{j}(t)}{d t} & =\sum_{i=1}^{n-1} n \mathbf{a}_{i}^{j} B_{i-1, n-1}(t)-\sum_{i=0}^{n-1} n \mathbf{a}_{i}^{j} B_{i, n-1}(t) \\
& =\sum_{i=0}^{n-1} n \mathbf{a}_{i+1}^{j} B_{i, n-1}(t)-\sum_{i=0}^{n-1} n \mathbf{a}_{i}^{j} B_{i, n-1}(t) \\
& =\sum_{i=0}^{n-1} B_{i, n-1}(t) n\left\{\mathbf{a}_{i+1}^{j}-\mathbf{a}_{i}^{j}\right\} .
\end{aligned}
$$

Now, we specify the procedure of derivation of (10) from (9). By (6), we have

$$
\begin{aligned}
\mathbf{v}_{j}(t)= & \left(\begin{array}{l}
n \\
0
\end{array}\right) \mathbf{a}_{0}^{j} \frac{1}{h^{n}}\left(t_{j}-t\right)^{n} \\
& +\cdots+\left(\begin{array}{l}
n \\
n
\end{array}\right) \mathbf{a}_{n}^{j} \frac{1}{h^{n}}\left(t-t_{j-1}\right)^{n}, \\
\mathbf{v}_{j+1}(t)= & \left(\begin{array}{c}
n \\
0
\end{array}\right) \mathbf{a}_{0}^{j+1} \frac{1}{h^{n}}\left(t_{j+1}-t\right)^{n} \\
& +\cdots+\left(\begin{array}{c}
n \\
n
\end{array}\right) \mathbf{a}_{n}^{j+1} \frac{1}{h^{n}}\left(t-t_{j}\right)^{n} ;
\end{aligned}
$$

by substituting $t=t_{j}$ into (A.5), one has

$$
\begin{aligned}
\mathbf{v}_{j}\left(t_{j}\right) & =\mathbf{a}_{n}^{j} \frac{1}{h^{n}}\left(t_{j}-t_{j-1}\right)^{n}, \\
\mathbf{v}_{j+1}\left(t_{j}\right) & =\mathbf{a}_{0}^{j+1} \frac{1}{h^{n}}\left(t_{j+1}-t_{j}\right)^{n} .
\end{aligned}
$$


TABLE 1: Exact and estimated values of $x_{1}(t)$ for Example 3.

\begin{tabular}{lccc}
\hline$t$ & Exact $x_{1}(t)$ & Present $x_{1}(t)$ & $x_{1}(t)$ in $[42]$ \\
\hline 0.00 & 0.000000 & 0.0000000000000000 & -0.000088 \\
0.05 & 0.000000 & 0.0000050777072400 & -0.000046 \\
0.10 & 0.000000 & 0.0000000000000000 & 0.000021 \\
0.15 & 0.000000 & -0.000253099630375 & 0.000083 \\
0.20 & 0.000000 & -0.000501121553000 & -0.000128 \\
0.25 & 0.000000 & $-1.06875 \times 10^{-11}$ & -0.000024 \\
0.30 & 0.001250 & 0.0019414196591000 & 0.001400 \\
0.35 & 0.005000 & 0.0057172621996375 & 0.004987 \\
0.40 & 0.011250 & 0.0116454806360000 & 0.011157 \\
0.45 & 0.020000 & 0.0199999999857500 & 0.019968 \\
0.50 & 0.031250 & 0.0310107172150000 & 0.031304 \\
0.55 & 0.044971 & 0.04479153044625000 & 0.045021 \\
0.60 & 0.061000 & 0.06099999990000000 & 0.060991 \\
0.65 & 0.079086 & 0.0791835285950000 & 0.079044 \\
0.70 & 0.098917 & 0.0989798441000000 & 0.098901 \\
0.75 & 0.120117 & 0.1201170006000000 & 0.120143 \\
0.80 & 0.142244 & 0.1422502585600000 & 0.142266 \\
0.85 & 0.164728 & 0.1647280007500000 & 0.164710 \\
0.90 & 0.186819 & 0.1868145712000000 & 0.186803 \\
0.95 & 0.207606 & 0.2076060001475000 & 0.207623 \\
1.00 & 0.226030 & 0.2260300002000000 & 0.226030 \\
\hline & & &
\end{tabular}

TABLE 2: Exact and estimated values of $x_{2}(t)$ for Example 3.

\begin{tabular}{lccc}
\hline$t$ & Exact $x_{2}(t)$ & Present $x_{2}(t)$ & $x_{2}(t)$ in $[42]$ \\
\hline 0.00 & 0.000000 & 0.000000000000000 & 0.001169 \\
0.05 & 0.050000 & 0.050000000000000 & 0.049923 \\
0.10 & 0.100000 & 0.099999999970000 & 0.100294 \\
0.15 & 0.150000 & 0.150424766127000 & 0.149740 \\
0.20 & 0.200000 & 0.200976855207000 & 0.199902 \\
0.25 & 0.250000 & 0.250636614672500 & 0.250170 \\
0.30 & 0.298229 & 0.298229000000000 & 0.298294 \\
0.35 & 0.345083 & 0.342083000067500 & 0.342098 \\
0.40 & 0.380313 & 0.380416662700000 & 0.380186 \\
0.45 & 0.411667 & 0.411748202343750 & 0.411593 \\
0.50 & 0.434896 & 0.434896000125000 & 0.435025 \\
0.55 & 0.448306 & 0.4482677054750000 & 0.448326 \\
0.60 & 0.448532 & 0.4485758408000000 & 0.448483 \\
0.65 & 0.432078 & 0.432134688390000 & 0.432080 \\
0.70 & 0.395846 & 0.395846000275000 & 0.395868 \\
0.75 & 0.337199 & 0.337199000906250 & 0.337171 \\
0.80 & 0.254052 & 0.254052000960000 & 0.254038 \\
0.85 & 0.145303 & 0.145637497343750 & 0.145354 \\
0.90 & 0.011316 & 0.011635894970000 & 0.011295 \\
0.95 & -0.145872 & -0.14587200166625 & -0.145924 \\
1.00 & -0.322405 & -0.32240500200000 & -0.322386 \\
\hline & & &
\end{tabular}

To preserve the continuity of the Bézier curves at the nodes, one needs to impose the condition $\mathbf{v}_{j}\left(t_{j}\right)=\mathbf{v}_{j+1}\left(t_{j}\right)$; so from (A.6), we have

$$
\mathbf{a}_{n}^{j}\left(t_{j}-t_{j-1}\right)^{n}=\mathbf{a}_{0}^{j+1}\left(t_{j+1}-t_{j}\right)^{n}
$$

TABLE 3: Exact and estimated values of $x(t)$ for Example 4.

\begin{tabular}{lcc}
\hline$t$ & Exact & Presented method \\
\hline 0.5 & 2.25 & 2.24991525903163 \\
1.0 & 4 & 4.00000000000000 \\
1.5 & 6.25 & 6.24995700258759 \\
2 & 9 & 9.00000000128046 \\
\hline
\end{tabular}

From (A.4), the first derivatives of $\mathbf{v}_{j}(t)$ and $\mathbf{v}_{j+1}(t)$ are, respectively,

$$
\begin{aligned}
\frac{d \mathbf{v}_{j}(t)}{d t}= & \sum_{i=0}^{n-1} B_{i, n-1}(t) n\left(\mathbf{a}_{i+1}^{j}-\mathbf{a}_{i}^{j}\right) \\
= & \sum_{i=0}^{n-1}\left(\begin{array}{c}
n-1 \\
i
\end{array}\right)\left(t_{j}-t\right)^{n-1-i}\left(t-t_{j-1}\right)^{i} \\
& \times \frac{1}{h^{n}}\left\{n\left(\mathbf{a}_{i+1}^{j}-\mathbf{a}_{i}^{j}\right)\right\} \\
= & \left(\begin{array}{c}
n-1 \\
0
\end{array}\right)\left\{n\left(\mathbf{a}_{1}^{j}-\mathbf{a}_{0}^{j}\right)\right\} \frac{1}{h^{n}}\left(t_{j}-t\right)^{n-1} \\
& +\cdots+\left(\begin{array}{l}
n-1 \\
n-1
\end{array}\right)\left\{n\left(\mathbf{a}_{n}^{j}-\mathbf{a}_{n-1}^{j}\right)\right\} \\
& \times \frac{1}{h^{n}}\left(t-t_{j-1}\right)^{n-1}, \\
\frac{d \mathbf{v}_{j+1}(t)}{d t}= & \sum_{i=0}^{n-1}\left(\begin{array}{c}
n-1 \\
i
\end{array}\right)\left(t_{j+1}-t\right)^{n-1-i}\left(t-t_{j}\right)^{i} \\
& \times \frac{1}{h^{n}}\left\{n\left(\mathbf{a}_{i+1}^{j+1}-\mathbf{a}_{i}^{j+1}\right)\right\} \\
= & \left(\begin{array}{c}
n-1 \\
0
\end{array}\right)\left\{n\left(\mathbf{a}_{1}^{j+1}-\mathbf{a}_{0}^{j+1}\right)\right\} \frac{1}{h^{n}}\left(t_{j+1}-t\right)^{n-1} \\
& +\cdots+\left(\begin{array}{l}
n-1 \\
n-1
\end{array}\right)\left\{n\left(\mathbf{a}_{n}^{j+1}-\mathbf{a}_{n-1}^{j+1}\right)\right\} \\
& \times \frac{1}{h^{n}}\left(t-t_{j}\right)^{n-1} .
\end{aligned}
$$

By substituting $t=t_{j}$ into (A.8), we have

$$
\begin{aligned}
& \frac{d \mathbf{v}_{j}\left(t_{j}\right)}{d t}=n\left(\mathbf{a}_{n}^{j}-\mathbf{a}_{n-1}^{j}\right) \frac{1}{h^{n}}\left(t_{j}-t_{j-1}\right)^{n-1}, \\
& \frac{d \mathbf{v}_{j+1}\left(t_{j}\right)}{d t}=n\left(\mathbf{a}_{1}^{j+1}-\mathbf{a}_{0}^{j+1}\right) \frac{1}{h^{n}}\left(t_{j+1} t_{j}\right)^{n-1},
\end{aligned}
$$

and to preserve the continuity of the first derivative of Bézier curves at nodes, by equalizing (A.9), we have

$$
\left(\mathbf{a}_{n}^{j}-\mathbf{a}_{n-1}^{j}\right)\left(t_{j}-t_{j-1}\right)^{n-1}=\left(\mathbf{a}_{1}^{j+1}-\mathbf{a}_{0}^{j+1}\right)\left(t_{j+1}-t_{j}\right)^{n-1},
$$

where it shows the equality (10). 
TABLE 4: Exact and estimated values of $x(t)$ for Example 5.

\begin{tabular}{lccccc}
\hline$t$ & Exact & Presented method & Method in [40] & Error of presented method & Error of the method in [40] \\
\hline 0.5 & 1.10468992792789 & 1.10468992817860 & 1.1451 & $2.50709000000000 \times 10^{-10}$ & $1.2232 \times 10^{-3}$ \\
1.0 & 1.53790187962670 & 1.53790188062000 & 1.5361 & $9.93297 \times 10^{-10}$ & $1.7685 \times 10^{-3}$ \\
1.4 & 1.93790187962670 & 1.93768171138582 & 1.9361 & $0.220168240883 \times 10^{-3}$ & $1.7685 \times 10^{-3}$ \\
1.5 & 2.03790187962670 & 2.03790188078453 & 2.0362 & $1.157827 \times 10^{-9}$ & $1.6125 \times 10^{-3}$ \\
2.0 & 2.53790187962670 & 2.53790188032000 & 2.5870 & $6.93297 \times 10^{-10}$ & $4.9096 \times 10^{-2}$ \\
\hline
\end{tabular}

TABLE 5: Exact and estimated values of $x(t)$ for Example 6.

\begin{tabular}{lccccc}
\hline$t$ & Exact & Presented method & Method in [40] & Error of presented method & Error of the method in [40] \\
\hline 1.0 & 0.25 & 0.250000000017634 & 0.250013 & $1.7634 \times 10^{-11}$ & $1.28346 \times 10^{-5}$ \\
2.0 & 0.0 & 0.0 & $5.26486 \times 10^{-7}$ & 0.0 & $5.26486 \times 10^{-7}$ \\
\hline
\end{tabular}

TABLE 6: Exact and estimated values of $x(t)$ for Example 8 .

\begin{tabular}{lcccc}
\hline$t$ & Exact & Presented method & Error Of presented method & Error of the method in [43] \\
\hline 0.2 & 0.04 & 0.0400000000049152 & $4.9152 \times 10^{-12}$ & $1.73 \times 10^{-6}$ \\
0.4 & 0.16 & 0.1600000000193540 & $1.9354 \times 10^{-11}$ & $1.10 \times 10^{-5}$ \\
0.6 & 0.36 & 0.3600000000221180 & $2.2118 \times 10^{-11}$ & $1.26 \times 10^{-4}$ \\
0.8 & 0.64 & 0.6400000000073730 & $7.373 \times 10^{-12}$ & $7.07 \times 10^{-4}$ \\
\hline
\end{tabular}

\section{Conflict of Interests}

The authors declare that they have no conflict of interests regarding publication of this paper.

\section{Acknowledgment}

The authors would like to thank the anonymous reviewers for their careful reading, constructive comments, and nice suggestions which have improved the paper very much.

\section{References}

[1] G. Adomian and R. Rach, "Nonlinear stochastic differential delay equations," Journal of Mathematical Analysis and Applications, vol. 91, no. 1, pp. 94-101, 1983.

[2] J. Baranowski, "Legendre polynomial approximations of time delay systems," in Proceedings of the 12th International PhD Workshop, p. 2326, 2010.

[3] F. Maghami Asl and A. G. Ulsoy, "Analysis of a system of linear delay differential equations," Journal of Dynamic Systems, Measurement and Control, Transactions of the ASME, vol. 125, no. 2, pp. 215-223, 2003.

[4] X. T. Wang, "Numerical solution of delay systems containing inverse time by hybrid functions," Applied Mathematics and Computation, vol. 173, no. 1, pp. 535-546, 2006.

[5] J. K. Hale and S. M. V. Lunel, Introduction to Functional Differential Equations, Springer, New York, NY, USA, 1993.

[6] S. I. Niculescu, Delay Effects on Stability: a Robust Control Approach, Springer, New York, NY, USA, 2001.

[7] D. H. Eller and J. I. Aggarwal, "Optimal control of linear timedelay systems," IEEE Transactions on Automatic Control, vol. 14, no. 14 , pp. 678-687, 1969.

[8] L. Göllmann, D. Kern, and H. Maurer, "Optimal control problems with delays in state and control variables subject to mixed control-state constraints," Optimal Control Applications and Methods, vol. 30, no. 4, pp. 341-365, 2009.

[9] N. N. Krasovskii, "Optimal processes in systems with time lags," in Proceedings of the 2nd International Conference of International Federation of Automatic Control, Basel, Switzerland, 1963.

[10] R. Loxton, K. L. Teo, and V. Rehbock, "An optimization approach to state-delay identification," IEEE Transactions on Automatic Control, vol. 55, no. 9, pp. 2113-2119, 2010.

[11] C. Wu, K. L. Teo, R. Li, and Y. Zhao, "Optimal control of switched systems with time delay," Applied Mathematics Letters, vol. 19, no. 10, pp. 1062-1067, 2006.

[12] G. L. Kharatishivili, "The maximum principle in the theory of optimal processes with time lags," Doklady Akademii Nauk SSSR, vol. 136, no. 1, 1961.

[13] M. N. Oguztoreli, "A time optimal control problem for systems described by differential difference equations," SIAM Journal of Control, vol. 1, no. 3, pp. 290-310, 1963.

[14] J. P. LaSalle, “The time optimal control problem," in Contributions to the Theory of Nonlinear Oscillations, vol. 5, pp. 1-24, Princeton University Press, Princeton, NJ, USA, 1960.

[15] N. N. Krasovskii, "On the analytic construction of an optimal control in a system with time lags," Prikladnaya Matematika $i$ Mekhanika, vol. 26, no. 1, pp. 50-67, 1962.

[16] D. W. Ross, Optimal control of systems described by differential difference equations [Ph.D. thesis], Department of Electrical Energy, Stanford University, Stanford, Calif, USA, 1968.

[17] M. Basin and J. Perez, "An optimal regulator for linear systems with multiple state and input delays," Optimal Control Applications and Methods, vol. 28, no. 1, pp. 45-57, 2007.

[18] M. Basin and J. Rodriguez-Gonzalez, "Optimal control for linear systems with multiple time delays in control input," IEEE Transactions on Automatic Control, vol. 51, no. 1, pp. 91-97, 2006.

[19] M. Heinkenschloss, "A time-domain decomposition iterative method for the solution of distributed linear quadratic optimal control problems," Journal of Computational and Applied Mathematics, vol. 173, no. 1, pp. 169-198, 2005. 
[20] H. Juddu, "Spectral method for constrained linear-quaratic optimal control," Mathematics Computers in Simulation, vol. 58, pp. 159-169, 2002.

[21] R. Winkel, "Generalized bernstein polynomials and Bézier curves: an application of umbral calculus to computer aided geometric design," Advances in Applied Mathematics, vol. 27, no. 1, pp. 51-81, 2001.

[22] J. Zheng, T. W. Sederberg, and R. W. Johnson, "Least squares methods for solving differential equations using Bézier control points," Applied Numerical Mathematics, vol. 48, no. 2, pp. 237$252,2004$.

[23] K. Harada and E. Nakamae, "Application of the Bézier curve to data interpolation," Computer-Aided Design, vol. 14, no. 1, pp. $55-59,1982$.

[24] G. Nürnberger and F. Zeilfelder, "Developments in bivariate spline interpolation," Journal of Computational and Applied Mathematics, vol. 121, no. 1, pp. 125-152, 2000.

[25] M. Evrenosoglu and S. Somali, "Least squares methods for solving singularly perturbed two-point boundary value problems using Bézier control points," Applied Mathematics Letters, vol. 21, no. 10, pp. 1029-1032, 2008.

[26] J. V. Beltran and J. Monterde, "Bézier solutions of the wave equation," in Computational Science and Its ApplicationsICCSA 2004, vol. 3044 of Lecture Notes in Computer Science, pp. 631-640, 2004.

[27] R. Cholewa, A. J. Nowak, R. A. Bialecki, and L. C. Wrobel, "Cubic Bezier splines for BEM heat transfer analysis of the 2D continuous casting problems," Computational Mechanics, vol. 28, no. 3-4, pp. 282-290, 2002.

[28] B. Lang, "The synthesis of wave forms using Bézier curves with control point modulation," in Proceedings of the 2nd CEMS Research Student Conference, Morgan Kaufamann, 2002.

[29] A. T. Layton and M. Van de Panne, "A numerically efficient and stable algorithm for animating water waves," The Visual Computer, vol. 18, no. 1, pp. 41-53, 2002.

[30] M. Gachpazan, "Solving of time varying quadratic optimal control problems by using Bézier control points," Computational and Applied Mathematics, vol. 30, no. 2, pp. 367-379, 2011.

[31] F. Ghomanjani and M. H. Farahi, "The Bézier control points method for solving delay differential equation," Intelligent Control and Automation, vol. 3, no. 2, pp. 188-196, 2012.

[32] F. Ghomanjani, M. H. Farahi, and M. Gachpazan, "Bézier control points method to solve constrained quadratic optimal control of time varying linear systems," Computational and Applied Mathematics, vol. 31, no. 3, p. 124, 2012.

[33] F. Ghomanjani, M. H. Farahi, and A. V. Kamyad, "Numerical solution of some linear optimal control systems with pantograph delays," IMA Journal of Mathematical Control and Information, 2013.

[34] F. Ghomanjani, M. H. Farahi, and M. Gachpazan, "Optimal control of time-varying linear delay systems based on the Bezier curves," Computational and Applied Mathematics, 2013.

[35] C.-H. Chu, C. C. L. Wang, and C.-R. Tsai, "Computer aided geometric design of strip using developable Bézier patches," Computers in Industry, vol. 59, no. 6, pp. 601-611, 2008.

[36] G. E. Farin, Curve and Surfaces for Computer Aided Geometric Design, Academic Press, New York, NY, USA, 1st edition, 1988.

[37] Y. Q. Shi and H. Sun, Image and Video Compression for Multimedia Engineering, CRC, 2000.

[38] A. Kilıçman, "On the matrix convolutional products and their applications," AIP Conference Proceedings, vol. 1309, pp. 607622, 2010
[39] A. Kılıçman and Z. Al Zhour, "Kronecker operational matrices for fractional calculus and some applications," Applied Mathematics and Computation, vol. 187, no. 1, pp. 250-265, 2007.

[40] I. Győri, F. Hartung, and J. Turi, "On numerical solutions for a class of nonlinear delay equations with time-and statedependent delays," in Proceedings of the World Congress of Nonlinear Analysts, pp. 1391-1402, New York, NY, USA, 1996.

[41] W. Rudin, Principles of Mathematical Analysis, McGraw-Hill, 1986.

[42] C. Hwang and M.-Y. Chen, "Analysis of time-delay systems using the Galerkin method," International Journal of Control, vol. 44, no. 3, pp. 847-866, 1986.

[43] O. A. Taiwo and O. S. Odetunde, "On the numerical approximation of delay differential equations by a decomposition method," Asian Journal of Mathematics and Statitics, vol. 3, no. 4, pp. 237243, 2010.

[44] H. Prautzsch, W. Boehm, and M. Paluszny, Bézier and B-Spline Techniques, Springer, 2001. 


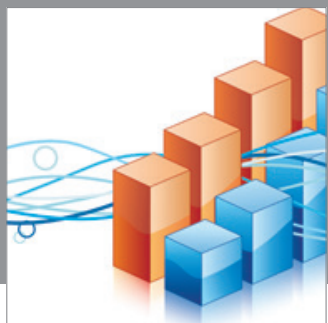

Advances in

Operations Research

mansans

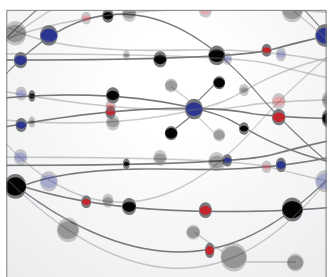

The Scientific World Journal
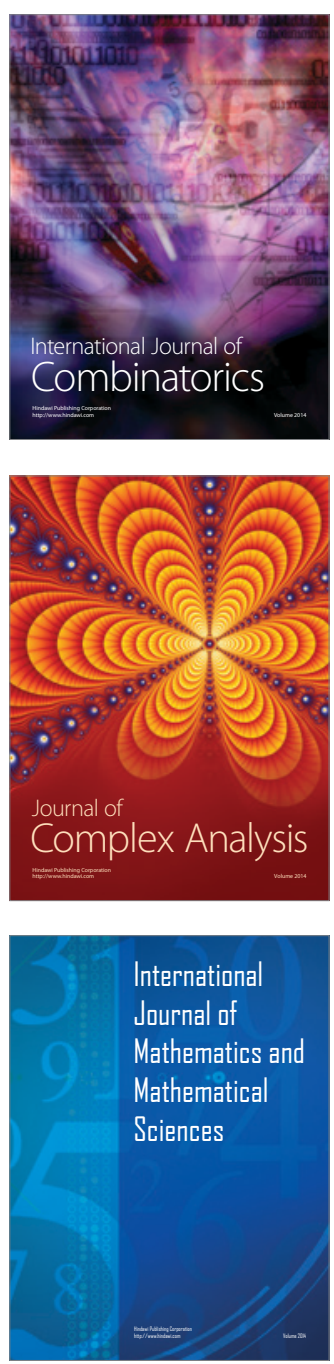
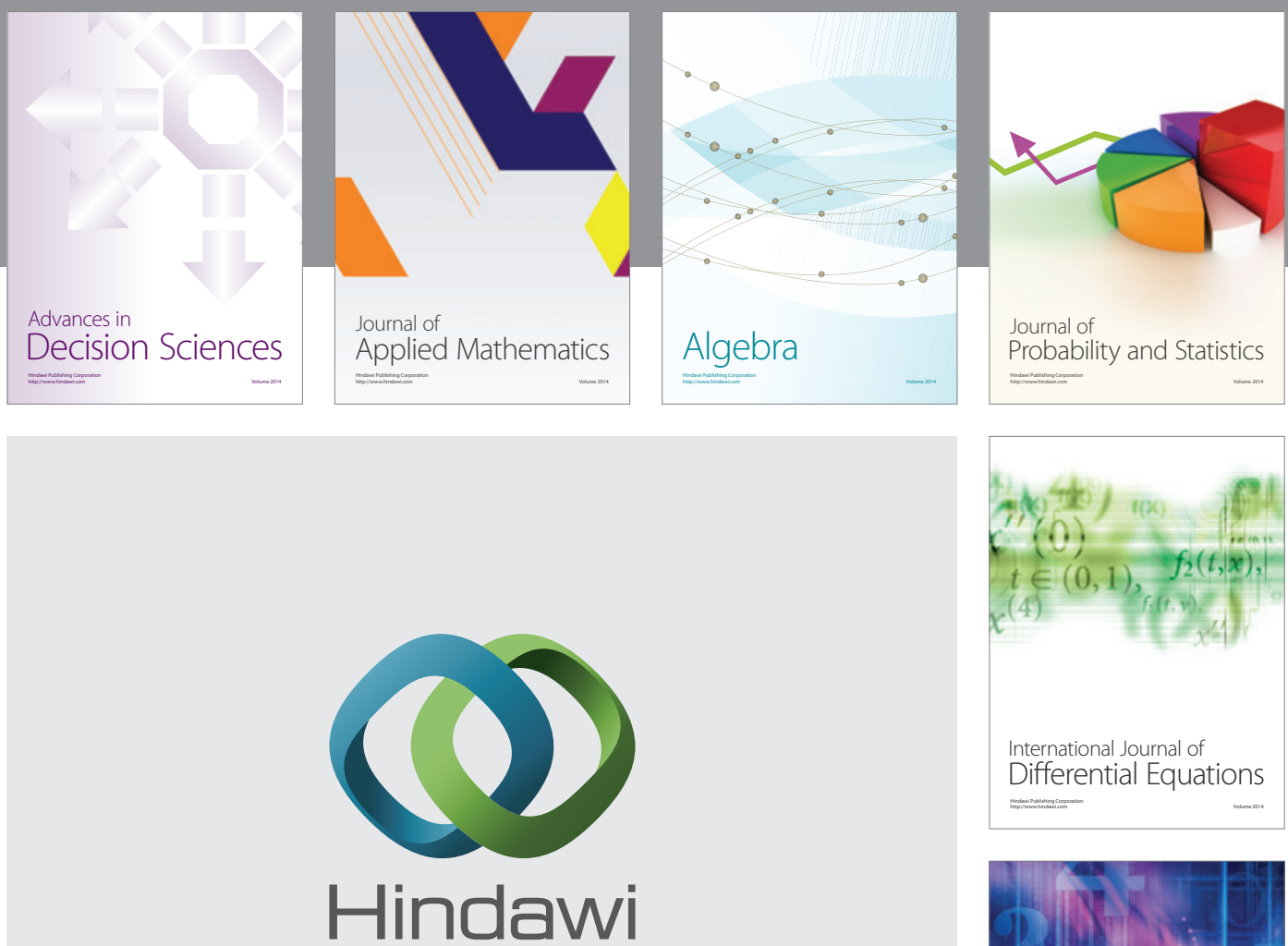

Submit your manuscripts at http://www.hindawi.com
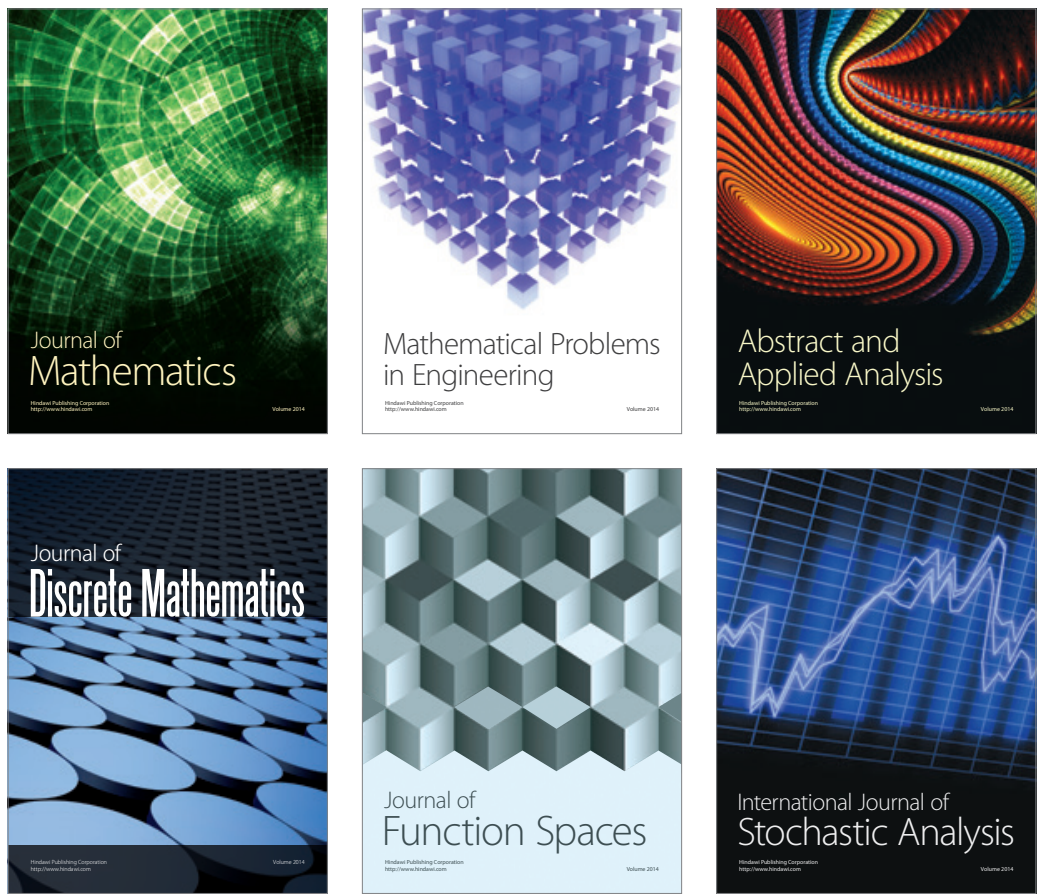

Journal of

Function Spaces

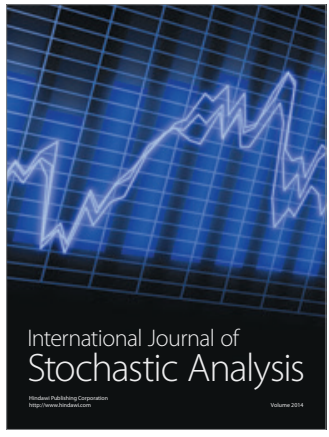

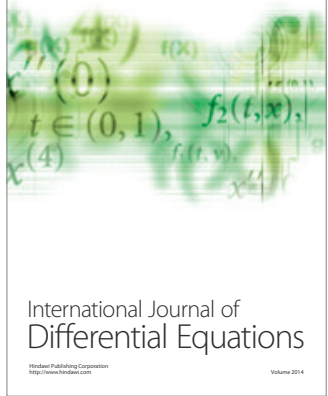
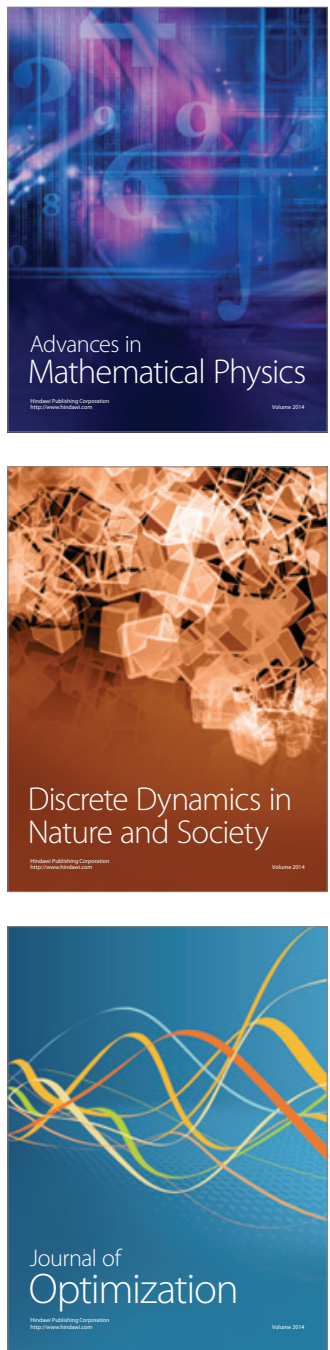